TRANSACTIONS OF THE

AMERICAN MATHEMATICAL SOCIETY

Volume 354, Number 6, Pages 2207-2241

S 0002-9947(02)02975-6

Article electronically published on February 14, 2002

\title{
PARTIAL REGULARITY FOR THE STOCHASTIC NAVIER-STOKES EQUATIONS
}

\author{
FRANCO FLANDOLI AND MARCO ROMITO
}

\begin{abstract}
The effects of random forces on the emergence of singularities in the Navier-Stokes equations are investigated. In spite of the presence of white noise, the paths of a martingale suitable weak solution have a set of singular points of one-dimensional Hausdorff measure zero. Furthermore statistically stationary solutions with finite mean dissipation rate are analysed. For these stationary solutions it is proved that at any time $t$ the set of singular points is empty. The same result holds true for every martingale solution starting from $\mu_{0}$-a.e. initial condition $u_{0}$, where $\mu_{0}$ is the law at time zero of a stationary solution. Finally, the previous result is non-trivial when the noise is sufficiently non-degenerate, since for any stationary solution, the measure $\mu_{0}$ is supported on the whole space $H$ of initial conditions.
\end{abstract}

\section{INTRODUCTION}

The possible emergence of singularities is one of the main open questions on the three-dimensional Navier-Stokes equations.

This problem is fundamental from the viewpoint of mathematical physics, but it is also related to the understanding of the evolution of $3 \mathrm{D}$ structures like thin vortex filaments, the intensity of the vortex stretching, and the transfer rate of energy from larger to smaller scales. So it is relevant for the physical understanding of turbulence in fluids.

One of the most notable characteristics of the turbulence is the absence of a typical scale of length. This is the basis from which Kolmogorov [13] started his celebrated analysis of homogeneous turbulence. Kolmogorov made an analysis of the energy spectra and of their analytic structure. A self-similar picture of these quantities arises, where the energy is transferred from large scales to smaller scales. This phenomenon reproduces itself from scale to scale, giving life to the so-called cascade of energy. But the Kolmogorov analysis does not consider the geometry of turbulence, which can be suggested by this picture.

In his pioneering paper [15] on the Navier-Stokes equations, J. Leray first noticed that a break in regularity can be produced by the following self-similar solutions:

$$
v(t, x)=\frac{1}{\sqrt{T-t}} V\left(\frac{x}{\sqrt{T-t}}\right),
$$

Received by the editors January 11, 2001 and, in revised form, July 21, 2001.

2000 Mathematics Subject Classification. Primary 76D05; Secondary 35A20, 35R60.

Key words and phrases. Navier-Stokes equations, singularities, partial regularity, suitable weak solutions, martingale solutions, stationary solutions. 
where $V$ solves a suitable second-order elliptic system. These solutions are invariant for the scaling

$$
\lambda \rightarrow \lambda v\left(\lambda^{2} t, \lambda x\right)
$$

In general it should not be expected, as it is suggested in Leray, that a solution having a singularity is self-similar (as a matter of facts, Necas and others [18] proved that no such solution exists). What is possible is that, where a singularity arises, the local profile of the energy or of some related quantities are designed by the Leray's scaling.

Mandelbrot, in [16] and [17, proposed a fractal homogeneous turbulence. This means that Kolmogorov's description applies only where the energy concentrates. Then the local self-similarity of the singularities forces the set where the energy accumulates to be a fractal set. Mandelbrot suggests that this claim can be considered as the geometrical counterpart of the self-similarity of the energy spectra of Kolmogorov theory.

Inspired by these considerations of Mandelbrot, Scheffer gave an upper bound for the fractal dimension of the set of singularities (see [21]-24]). But it is the paper by Caffarelli, Kohn and Nirenberg that shed the most light on this topic. In their work they combine the considerations on self-similarity and on the Leray scaling (1.1) with the control of energy transfer to give the best known result (and probably the best possible with their approach). In view of the scaling invariance, their estimates are given in terms of dimensionless quantities, such as the local rate of dissipation

$$
\delta(r)=\frac{1}{r} \int_{t-r^{2}}^{t} \int_{B_{r}(x)}|\nabla u|^{2},
$$

or the local flow of kinetic energy

$$
G(r)=\frac{1}{r^{2}} \int_{t-r^{2}}^{t} \int_{B_{r}(x)}|u|^{3} .
$$

These are dimensionless quantities in the sense that they are invariant under Leray's scaling transformation (1.1). Moreover these quantities are local, in order to have a localised control of the energy transfer through the scales.

It is not clear if the set of singularities is empty or not. When the viscosity is zero, some numerical investigations (see for instance Bell and Marcus [1, Chorin 3], Grauer and Sideris [1], Kerr [12], and others) support the belief that the set of singularities is not empty, but in the viscous case the answer is even less clear. From the mathematical point of view, the counterexamples presented by Scheffer in [25]-27] show at least that the approach of the balance of energy is not sufficient to prove that no singularities arise.

The original motivation of the research presented in this paper has been to investigate the effects of random forces on the emergence of singularities.

Different interpretations can be given to these terms. A random force can represent all those phenomena that are usually neglected where the system is stable. A random solution of the Navier-Stokes equations can take into account that flows with large Reynolds number are chaotic and sensitive to microscopic perturbations. Sources of perturbations can be the roughness of the boundary, or the effects of the environment external to the system, that is, acoustic waves, etc. 
A priori it could be guessed that white noise body forces introduce further irregularity and help the creation of singularities. On the contrary, it could be believed that noise breaks the formation and development of those coherent structures (like thin vortex tubes) that eventually could be responsible for the emergence of singularities. Another reason for interest in this approach is that eventually the external noise can be something different from the intrinsic fluctuations of the deterministic turbulent fluids, but the understanding of the effect of noise could give a hint in the latter case too.

The problem of singularities will not be studied for an individual solution, but for a stochastic process solution of the Navier-Stokes equations. Until now it has not been possible to understand this problem completely. However, some partial results can be proved. In fact the one-dimensional Hausdorff measure in space and time of the set of singularities is still zero, in spite of the presence of white noise or of deterministic distributional-in-time forcing terms.

Something more can be said. Having in mind the research of Lanford [14, Siegmund-Schultze [30, and other authors who prove existence of solutions to difficult dynamical problems for a.e. initial condition with respect to some timeinvariant or space-homogeneous measure, the study is focused on the singularities of the stationary suitable weak solutions. The stationarity has to be understood in a probabilistic sense; thus these solutions may describe a fluid in a turbulent regime. It is proved that at every time the set of singularities is empty with probability one. The result derives from the control of the mean dissipation rate

$$
\mathbf{E} \frac{1}{|D|} \int_{D} \nu|\nabla u|^{2}
$$

(which has the physical dimensions of the rate of energy dissipation $\epsilon$ of the Kolmogorov theory). In other words, at every time it is not possible to see the singularities: only a negligible set of paths may have singularities at a fixed time.

In a sense, this fact means that singularities do not arise in the stationary regime, confirming some claims, made on physical grounds, that the rate of vortex stretching and the very strong vorticity intensification (and possibly blow-up) typical of 3D fluids in the transient regime (when energy is transferred to small scales to form the inertial range), may decrease in the long time regime (see for instance Chorin [4]).

The paper is organised as follows. In the second section we give the definition of suitable weak solution, both deterministic and martingale, as well as the definition of stationary solution. A formal derivation of the local energy inequality is also presented. The third section is devoted to the presentation of the main results of the paper.

The partial regularity result is proved in the fourth and fifth sections. First it is shown that the local rate of dissipation controls the flow of energy. Then it is proved that the control of the flow of energy gives the local regularity.

The sixth section contains the proof of the claims on stationary solutions. Finally, in the appendix all the auxiliary results concerning the Stokes system are proved.

1.1. Some notations. In this section we set some notations that will be used throughout the paper. Define the space

$$
H=\left\{u: D \rightarrow \mathbf{R}^{3}\left|u \in\left(L^{2}(D)\right)^{3}, \operatorname{div} u=0, u \cdot n\right|_{\partial D}=0\right\},
$$


where $n$ is the outer normal to $\partial D$ (see for example Temam [33]), and the space

$$
V=\left\{u \in\left(H^{1}(D)\right)^{3}|\operatorname{div} u=0, u|_{\partial D}=0\right\} .
$$

The $L^{2}$-norm of elements of $H$ will be denoted by $|\cdot|$ and the $H^{1}$-norm of elements of $V$ will be denoted by $\|\cdot\|$. If the space $H$ is identified with its dual and the space $H^{\prime}$ is identified with a subspace of $V^{\prime}$, then

$$
V \subset H \subset V^{\prime} .
$$

The operator $A: D(A) \subset H \rightarrow H$ is defined as $A u=-\mathcal{P} \triangle u$, where $\mathcal{P}$ is the orthogonal projection from $L^{2}(D)^{3}$ onto $H$ and $D(A)=H^{2}(D)^{3} \cap V$. The operator $A$ is positive self-adjoint with compact resolvent (see for example Temam [35]). The eigenvalues of $A$ are denoted by $0<\lambda_{1} \leq \lambda_{2} \leq \ldots$, and $e_{1}, e_{2}, \ldots$ is a corresponding complete orthonormal system of eigenvectors.

Moreover, if the dual space of $D(A)$ is denoted by $D\left(A^{-1}\right)$, then identifications as above give the dense continuous inclusions

$$
D(A) \subset V \subset H \subset V^{\prime} \subset D\left(A^{-1}\right) .
$$

The fractional powers $A^{\alpha}$ of $A, \alpha \geq 0$, are simply defined by

$$
A^{\alpha} x=\sum_{i=1}^{\infty} \lambda_{i}^{\alpha}\left\langle x, e_{i}\right\rangle e_{i}
$$

with domain

$$
D\left(A^{\alpha}\right)=\left\{x \in H \mid\|x\|_{D\left(A^{\alpha}\right)}<\infty\right\}
$$

where

$$
\|x\|_{D\left(A^{\alpha}\right)}^{2}=\sum_{i=1}^{\infty} \lambda_{i}^{2 \alpha}\left\langle x, e_{i}\right\rangle^{2}=\left|A^{\alpha} x\right|^{2} .
$$

The space $D\left(A^{\alpha}\right)$ is an Hilbert space with the inner product

$$
\langle x, y\rangle_{D\left(A^{\alpha}\right)}=\left\langle A^{\alpha} x, A^{\alpha} y\right\rangle, \quad x, y \in D\left(A^{\alpha}\right) .
$$

Notice that

$$
D\left(A^{\alpha}\right) \subset H^{2 \alpha}(D)
$$

\section{Suitable Weak solutions}

Consider a viscous incompressible homogeneous Newtonian fluid in the bounded open domain $D \subset \mathbf{R}^{3}$ (with regular boundary), described by the Navier-Stokes equations

$$
\begin{gathered}
\frac{\partial u}{\partial t}-\nu \Delta u+(u \cdot \nabla) u+\nabla P=f+\frac{\partial g}{\partial t}, \\
\operatorname{div} u=0,
\end{gathered}
$$

where $u$ is the velocity field, $P$ is the pressure field and $\nu$ is the kinematic viscosity. For the sake of simplicity it will be assumed $\nu=1$, since its value is not relevant in the present setting. The term $\partial_{t} g$ represents a rapidly fluctuating force, and the function $g$ will be either a deterministic function, not differentiable in time, with a suitable regularity, or a Brownian motion $\left(B_{t}\right)_{t \geq 0}$ in a certain Hilbert space, so that $\dot{B}_{t}$ will be a white noise in such space. 
2.1. Formal derivation of the local energy inequality. Prior to giving the rigorous definition of suitable weak solutions, we argue formally, without precise assumptions on the data and the properties of the solutions, with the aim of devising the correct energy inequality.

The local energy inequality should involve a term like $\iint \partial_{t} g \cdot u \varphi$, where $\varphi$ is a test function. Since we do not assume any differentiability in time of $g$ (so that also $u$ is not expected to be differentiable in time), the meaning of this term is not clear. To overcome this difficulty we introduce new variables

$$
v=u-z, \quad \pi=P-Q,
$$

where the pair $(z, Q)$ solves the following Stokes equation:

$$
\begin{gathered}
\frac{\partial z}{\partial t}-\triangle z+\nabla Q=f+\frac{\partial g}{\partial t}, \\
\operatorname{div} z=0, \\
z(0)=0,
\end{gathered}
$$

in $[0, T] \times D$. The new pair $(v, \pi)$ solves the modified Navier-Stokes equations

$$
\begin{gathered}
\frac{\partial v}{\partial t}-\Delta v+((v+z) \cdot \nabla)(v+z)+\nabla \pi=0 \\
\operatorname{div} v=0
\end{gathered}
$$

where the white noise term has disappeared. While $P$ satisfies

$$
\triangle P=\sum_{i, j=1}^{3} \frac{\partial^{2}}{\partial x_{i} \partial x_{j}}\left(u_{i} u_{j}\right)
$$

the auxiliary variable $\pi$ satisfies

$$
\triangle \pi=\sum_{i, j=1}^{3} \frac{\partial^{2}}{\partial x_{i} \partial x_{j}}\left((v+z)_{i}(v+z)_{j}\right)
$$

The pair $(v, \pi)$ will be asked to satisfy a pathwise local energy inequality. This inequality can be obtained formally in the following way. If $\varphi \geq 0$ is a smooth function with compact support in $(0, T] \times D$, then

$$
\int_{0}^{t} \int_{D}\left(\frac{\partial v}{\partial t}-\Delta v+((v+z) \cdot \nabla)(v+z)+\nabla \pi\right) \cdot v \varphi=0
$$

Hence integration by parts gives

$$
\begin{aligned}
& \frac{1}{2} \int_{0}^{t} \int_{D} \frac{\partial|v|^{2}}{\partial t} \varphi+\frac{1}{2} \int_{0}^{t} \int_{D} \nabla|v|^{2} \cdot \nabla \varphi+\int_{0}^{t} \int_{D} v \varphi \cdot((v+z) \cdot \nabla) z \\
& \quad+\int_{0}^{t} \int_{D} \varphi|\nabla v|^{2}=\frac{1}{2} \int_{0}^{t} \int_{D}((v+z) \cdot \nabla)|v|^{2} \varphi+\int_{0}^{t} \int_{D} \pi v \cdot \nabla \varphi
\end{aligned}
$$


which implies

$$
\begin{aligned}
& \frac{1}{2} \int_{D}|v(t)|^{2} \varphi+\int_{0}^{t} \int_{D} \varphi|\nabla v|^{2} \\
& =\frac{1}{2} \int_{0}^{t} \int_{D}|v|^{2}\left(\frac{\partial \varphi}{\partial t}+\triangle \varphi\right)+\frac{1}{2} \int_{0}^{t} \int_{D}|v|^{2}((v+z) \cdot \nabla \varphi) \\
& \quad+\int_{0}^{t} \int_{D} \varphi z \cdot((v+z) \cdot \nabla) v+\int_{0}^{t} \int_{D}(v \cdot z)((v+z) \cdot \nabla \varphi) \\
& \quad+\int_{0}^{t} \int_{D} \pi v \cdot \nabla \varphi
\end{aligned}
$$

This formal computation motivates the definitions of the following sections.

2.2. Regularity for the Stokes system. As we have seen in the previous section, a weak solution $u$ is split as the sum of a nonlinear term $v$ plus a linear part $z$ which contains the body forces. In order to state the definitions of suitable weak solutions and the main results, we have to point out the assumptions on $f$ and $g$ that imply the regularity of $z$ needed in those statements.

The proof of the claims of this section can be found in Appendix

We consider equation (2.3) as a deterministic equation. When $g$ is a Brownian motion $\left(B_{t}\right)_{t \geq 0}$, its typical trajectory $B_{t}(\omega)$ fulfils certain regularity properties and, for such values of $\omega$, we consider equation (2.3) as a deterministic equation driven by $f+\partial_{t} B_{t}(\omega)$. So, for our purposes, it is sufficient to consider the deterministic case. See the additional remarks below on the stochastic case.

Lemma 2.1. Let $f \in L_{\text {loc }}^{p}([0, \infty) \times D) \cap L_{\text {loc }}^{2}([0, \infty) ; H)$ for some $p>2$, and let $g \in C^{\frac{1}{2}-\varepsilon}\left([0, T] ; D\left(A^{\beta}\right)\right)$ for some $\beta>\varepsilon>0$. Then there exist a unique vector field $z$ and a scalar field $Q$ (unique up to a constant), solution of equation (2.3), such that $z(0)=0$,

$$
z \in L_{\mathrm{loc}}^{\infty}([0, \infty) ; H) \cap L_{\mathrm{loc}}^{2}([0, \infty) ; V) \cap L_{\mathrm{loc}}^{\infty}\left([0, \infty) ; L_{\mathrm{loc}}^{q}(D)\right)
$$

for some $q>6$, and

$$
Q \in L_{\mathrm{loc}}^{5 / 3}((0, T) \times D) .
$$

Moreover, at each point $(t, x) \in(0, \infty) \times D$,

$$
\lim _{r \rightarrow 0} \frac{1}{r} \int_{t-r^{2}}^{t+r^{2}} \int_{B_{r}(x)}|\nabla z|^{2} d x d t=0 .
$$

Lemma 2.2. In addition to the assumptions of Lemma [2.1, assume that $f \in$ $L_{\text {loc }}^{p}([0, \infty) \times D)$ for some $p>\frac{5}{2}$, and $g \in C^{\frac{1}{2}-\varepsilon}\left([0, \infty) ; D\left(A^{\frac{1}{4}+\beta}\right)\right)$ for some $\beta>\varepsilon>0$. Then

$$
z \in L_{\mathrm{loc}}^{\infty}([0, \infty) \times D) .
$$

Remark 2.3. Given a Hilbert space $K$, we recall that a Brownian motion $\left(B_{t}\right)_{t \geq 0}$ in $K$ is a stochastic process with values in $K$, starting from the origin at time 0 , with independent Gaussian increments $B_{t}-B_{s}$, having zero mean and covariance $(t-s) \mathcal{O}$ for some nuclear operator $\mathcal{O}$ in $K$. See Da Prato and Zabczyk [5] for more details. With this definition in mind, let $\left(B_{t}\right)_{t \geq 0}$ be a Brownian motion, on a complete probability space $(\Omega, \mathcal{F}, \mathbf{P})$, with values in the Hilbert space $D\left(A^{\beta}\right)$, for 
some $\beta>0$. It can be proved that it has a modification, still denoted by $\left(B_{t}\right)_{t \geq 0}$, such that with probability one

$$
B \in C^{\alpha}\left([0, T] ; D\left(A^{\beta}\right)\right) \quad \text { for all } \alpha \in\left(0, \frac{1}{2}\right) .
$$

Therefore, for $\mathbf{P}$-a.e. $\omega \in \Omega$ the trajectory $B$. $(\omega)$ satisfies the assumptions of Lemma 2.1. So, given such $\omega \in \Omega$, the conclusions of the lemma hold for the corresponding trajectory of the stochastic process solution of the equation

$$
d z=(\triangle z+f-\nabla Q) d t+d B_{t} .
$$

In the same way, if $\left(B_{t}\right)_{t \geq 0}$ is a Brownian motion with values in $D\left(A^{\frac{1}{4}+\beta}\right)$ for some $\beta>0$, then for $\mathbf{P}$-a.e. $\omega \in \Omega$ the trajectory $B$. $(\omega)$ satisfies the assumptions of Lemma 2.2, and therefore for such $\omega \in \Omega$, the conclusions of the lemma hold.

Remark 2.4. Instead of using a Brownian motion $B$ with values in certain Hilbert spaces, we may adopt the following formalism. Consider the stochastic equation

$$
d z-\triangle z d t+\nabla Q d t=f d t+\sqrt{\mathcal{O}} d W,
$$

where $W$ is a cylindrical Wiener process in $H$ and $\mathcal{O}$ is a non-negative self-adjoint operator in $H$. For definitions and basic results, see [5]. We may now formulate assumptions on $\mathcal{O}$ and prove similar results. In particular, under the assumption

$$
\operatorname{Tr}\left[A^{\varepsilon} \mathcal{O} A^{\varepsilon}\right]<\infty \quad \text { for some } \varepsilon>0,
$$

we obtain $\mathbf{P}$-a.s. the result of Lemma 2.1, while under the assumption

$$
\operatorname{Tr}\left[A^{\frac{1}{4}+\varepsilon} \mathcal{O} A^{\frac{1}{4}+\varepsilon}\right]<\infty \quad \text { for some } \varepsilon>0,
$$

we obtain $\mathbf{P}$-a.s. the result of Lemma 2.2 .

2.3. The deterministic suitable weak solution. Let $T \leq \infty$ and let $f$ and $g$ be two distributions on $(0, T) \times D$, taking values in $\mathbf{R}^{3}$. It would be possible to unify $f+\partial_{t} g$ in a single distribution, but it is preferable to keep them separated in view of future assumptions. Under the assumptions of Lemma 2.1, we know that there exist

$$
z \in L^{\infty}\left(0, T ; L_{\mathrm{loc}}^{2}(D)\right) \cap L^{2}\left(0, T ; H_{\mathrm{loc}}^{1}(D)\right) \quad \text { and } \quad Q \in L_{\mathrm{loc}}^{5 / 3}((0, T) \times D)
$$

which solve the system $(2.3)$ in the sense of distributions on $(0, T) \times D$.

Definition 2.5. Given $f, g$ as in Lemma 2.1, let $(z, Q)$ be the solution of the Stokes problem. A suitable weak solution $(u, P)$ to the Navier-Stokes equations (2.1) is a pair

$$
u \in L^{\infty}\left(0, T ; L_{\mathrm{loc}}^{2}(D)\right) \cap L^{2}\left(0, T ; H_{\mathrm{loc}}^{1}(D)\right)
$$

and

$$
P \in L_{\mathrm{loc}}^{5 / 3}((0, T) \times D)
$$

such that the new variables $v=u-z$ and $\pi=P-Q$ satisfy the modified NavierStokes equations (2.4) in the sense of distributions over $(0, T) \times D$ and moreover 
satisfy the local energy inequality

$$
\begin{aligned}
& \int_{D}|v(t)|^{2} \varphi+2 \int_{0}^{t} \int_{D} \varphi|\nabla v|^{2} \\
& \leq \int_{0}^{t} \int_{D}|v|^{2}\left(\frac{\partial \varphi}{\partial t}+\Delta \varphi\right)+\int_{0}^{t} \int_{D}\left(|v|^{2}+2 v \cdot z\right)((v+z) \cdot \nabla \varphi) \\
& \quad+2 \int_{0}^{t} \int_{D} \varphi z \cdot((v+z) \cdot \nabla) v+\int_{0}^{t} \int_{D} 2 \pi v \cdot \nabla \varphi
\end{aligned}
$$

for every smooth function $\varphi: \mathbf{R} \times D \rightarrow \mathbf{R}, \varphi \geq 0$, with compact support in $(0, T] \times D$.

All the terms in the local energy inequality are well defined, since $\pi \in L^{5 / 3}$ and by the Sobolev inequality $v$ and $z$ are in $L^{p}$ for each $p \in[2,10 / 3)$.

Remark 2.6. We have chosen the solution $z$ of the Stokes equation with initial condition $z(0)=0$. The definition of suitable weak solution we have just given seems to depend on this choice. This is not true: the definition is independent of the splitting, see Section 2 of [19]

2.4. The martingale suitable weak solution. The martingale suitable weak solutions for the Navier-Stokes system are solutions of a stochastic differential equation driven by an additive noise which are almost surely suitable weak solutions in the sense of Definition 2.5 The term $g$ will be a Brownian motion $\left(B_{t}\right)_{t \geq 0}$, subject to the assumptions given in Remark 2.3 above, in such a way that the regularity of $z$ and $Q$ are assured.

Definition 2.7. A martingale suitable weak solution is a process $(u, P)$ defined on a stochastic basis $\left(\Omega, \mathcal{F},(\mathcal{F})_{t \geq 0}, \mathbf{P},\left(B_{t}\right)_{t \geq 0}\right)$, where $B$ is a Brownian motion adapted to the filtration with values in $D\left(A^{\beta}\right)$, such that

$$
\omega \in \Omega \rightarrow(u(\omega), P(\omega)) \in L^{2}(0, T ; H) \times L_{\text {loc }}^{5 / 3}((0, T) \times D)
$$

is a measurable mapping and such that there exists a set $\Omega_{0} \subset \Omega$ of full probability such that $(u(\cdot, \omega), P(\cdot, \omega))$ is a suitable weak solution in the sense of Definition 2.5 with respect to the forcing term $\partial_{t} B_{t}(\omega)$, for all $\omega \in \Omega_{0}$.

Let us explain the meaning of the last part of the definition. Since $B$ is a Brownian motion in $D\left(A^{\beta}\right)$, by Remark $\left[2.3\right.$ it has a.s. trajectories in $C^{\alpha}\left([0, T] ; D\left(A^{\beta}\right)\right)$ for all $\alpha \in\left(0, \frac{1}{2}\right)$. Then in the definition above we require that there exists a full measure set $\Omega_{0} \subset \Omega$ such that for every given $\omega \in \Omega_{0}$ the following statement is true: we have $B(\omega) \in C^{\alpha}\left([0, T] ; D\left(A^{\beta}\right)\right)$ for all $\alpha \in\left(0, \frac{1}{2}\right)$ and, denoting by $(z(\omega), Q(\omega))$ the solution of the deterministic Stokes system driven by $B(\omega)$, the pair $(u(\omega), P(\omega))$ satisfies all the conditions of Definition 2.5 with respect to these functions.

It is worth noticing that such solutions exist. A proof of this claim is given in [19. Note that a similar definition can be given also in the framework of Remark 2.4. where a cylindrical Wiener process $W_{t}$ is considered.

Remark 2.8. The concept of martingale solution is equivalent to the concept of statistical solution, as given by Foias, Temam, and others. In order to understand this claim, we introduce the precise setting.

Let $C_{0}([0,+\infty), H)$ be the set of all continuous functions which take value 0 in $t=0$ and let $\mathcal{S}$ be the subset of $L_{\mathrm{loc}}^{2}([0,+\infty) ; H) \times C_{0}([0,+\infty), H)$ of all 
suitable weak solutions in $(0, \infty) \times D$, that is, the set of all pairs $(u, B)$, where $B \in C^{\frac{1}{2}-\varepsilon}\left([0, T] ; D\left(A^{\beta}\right)\right)$ for some $\beta>\varepsilon>0$ and $u$ is a suitable weak solution (in the sense of Definition [2.5), in each time interval $[0, T]$, of the Navier-Stokes equation forced by $\partial_{t} B$. In this setting the pressure $P$ is treated as an auxiliary scalar field.

A metric is defined on $\mathcal{S}$ in the following way. If $\left(u^{1}, B^{1}\right)$ and $\left(u^{2}, B^{2}\right)$ are in $\mathcal{S}$,

$$
\begin{aligned}
d\left(\left(u^{1}, B^{1}\right),\left(u^{2}, B^{2}\right)\right)= & \sum_{n=1}^{\infty} 2^{-n}\left(1 \wedge \int_{0}^{n}\left|v^{1}-v^{2}\right|^{2} d t\right)^{1 / 2} \\
& +\sum_{n=1}^{\infty} 2^{-n}\left(1 \wedge \sup _{(0, n)}\left|B^{1}-B^{2}\right|\right)
\end{aligned}
$$

Let $C_{b}(\mathcal{S})$ be the space of all bounded real continuous functions on $\mathcal{S}$ with the uniform topology, let $\mathcal{B}$ be the Borel $\sigma$-algebra of $(\mathcal{S}, d)$, and let $M_{1}(\mathcal{S})$ be the set of all probability measures on $(\mathcal{S}, \mathcal{B})$.

A statistical suitable weak solution of Navier-Stokes equations (2.1) is simply a probability measure $\mu \in M_{1}(\mathcal{S})$. The classical case where $\left(B_{t}\right)_{t \geq 0}$ is a Brownian motion is recovered simply by assuming that the marginal law of $\mu$ on the second component of $\mathcal{S}$ is the law of the Brownian motion itself. It is not difficult to reinterpret this concept by means of stochastic processes $u, B$ satisfying the NavierStokes equations $\mathbf{P}$-a.s. (as well as the regularity conditions and the local energy inequality). To this purpose it is sufficient to consider the canonical processes defined on $\mathcal{S}$, under the law $\mu$. Vice versa, if we have a martingale solution $u$, the joint law of $u$ and the Brownian motion $B$ is a statistical suitable weak solution $\mu \in M_{1}(\mathcal{S})$ in the sense of the definition above.

2.5. The stationary solution. A stationary solution is a statistical solution, in the sense given above, which is invariant under the time-shift. In order to have an autonomous equation in the deterministic part, the mean forcing term $f \in H$ will be assumed independent of time. The time-shift will act on the increments of the Brownian motion in order to preserve the stationarity of its increments.

Let $\tau_{t}: \mathcal{S} \rightarrow \mathcal{S}(t \geq 0)$ be the time shift on $\mathcal{S}$, defined as

$$
\tau_{t}(u, B)(s)=(u(s+t), B(t+s)-B(t)) .
$$

The map $(t, u, B) \rightarrow \tau_{t}(u, B)$ is continuous from $[0, \infty) \times \mathcal{S}$ to $\mathcal{S}$. The induced mapping on $C_{b}(\mathcal{S})$, defined as

$$
\tau_{t} \phi(u, B)=\phi\left(\tau_{t}(u, B)\right),
$$

will be denoted again by $\tau_{t}$, and $\tau_{t} \mu$ will be the image measure of any $\mu \in M_{1}(\mathcal{S})$ under $\tau_{t}$, in the sense that

$$
\left\langle\tau_{t} \mu, \phi\right\rangle=\left\langle\mu, \tau_{t} \phi\right\rangle
$$

for each $\phi \in C_{b}(\mathcal{S})$.

Definition 2.9. A probability measure $\mu \in M_{1}(\mathcal{S})$ is time-stationary if $\tau_{t} \mu=\mu$ for all $t \geq 0$. We say that $\mu$ has finite mean dissipation rate if

$$
\int_{\mathcal{S}}\left[\int_{0}^{T} \int_{D}|\nabla u|^{2} d x d t\right] \mu(d(u, B))<\infty
$$

for all $T>0$. 
A proof of the existence of a stationary solution is given in [19], when the marginal measure of $\mu$ in the second component is the law of a Brownian motion. But we believe that this result may be proved also in the case when the marginal measure is the law of some other process with stationary increments, such as fractional Brownian motions, etc.

\section{Main Results}

Let us state the precise results. The main interest is in regular points for solutions of equation (2.1). A point $(t, x)$ is regular for a solution $u$ if there exists a neighbourhood of $(t, x)$ where $u$ is essentially bounded. The other points will be called singular. The set of singular points of $u$ will be denoted by $S(u)$.

The choice to consider such a definition for regular space-time points relies on a paper by Serrin (see [28] and also [29]) which proved that if a weak solution of the Navier-Stokes equations has locally a further summability (in particular, if it is bounded), then the solution is locally smooth in the space variable. That is, the only way a singularity of a solution can arise is by means of a blow-up of the velocity field; it cannot happen that some derivative of $u$ can be singular without a singularity of $u$.

In [20] a Serrin type result is proved for the pair $(v, \pi)$, a solution of equation (2.4). If the solution $v$ has a higher summability, then it is $C^{\infty}$ in the space variable (given regular data). Hence the definition of singular points makes sense in this case too.

Using the splitting $u=v+z$, the data $f$ and $g$ do not explicitly appear in the equation for $(v, \pi)$. It is then clear that, in several parts of the paper, the following strategy will be adopted: on one side some results on the singularities of $v$ will be established under certain regularity conditions on $(z, Q)$; on the other side the results of Section 2.2 identify assumptions on $f$ and $g$ that give such conditions on $(z, Q)$.

Although this splitting approach is motivated by the presence of the distribution $\partial_{t} g$, it gives at least one new result also in the case $g \equiv 0$. Indeed, in Caffarelli, Kohn and Nirenberg [2] the partial regularity result has been proved when $f \in L^{p}$ for some $p>\frac{5}{2}$. The procedure used here does not allow us to improve this result without changing the definition of regular point, but at least it leads to the following related result:

Proposition 3.1. Let $(u, P)$ be a suitable weak solution to system (2.1), in the sense of Definition 2.5 above. If

$$
g \equiv 0, \quad f \in L^{p}((0, T) \times D) \quad \text { for some } p \in\left(2, \frac{5}{2}\right],
$$

then $u(t) \in L_{\mathrm{loc}}^{\infty}(D)$ for a.e. $t \in[0, T]$, and there exists $D_{0} \subset D$ such that $\mathcal{H}^{1}\left(D_{0}\right)=$ 0 and for each $x \in D \backslash D_{0}$

$$
\sup _{t \in(0, T)}|u(t, x)|<\infty
$$

The first main result is

Theorem 3.2. Let

$$
f \in L^{p}((0, T) \times D) \cap L^{2}(0, T ; H)
$$


for some $p>\frac{5}{2}$ and

$$
g \in C^{\frac{1}{2}-\varepsilon}\left([0, T] ; D\left(A^{\frac{1}{4}+\delta}\right)\right)
$$

for some $\beta>\varepsilon>0$. Let $(u, P)$ be a suitable weak solution of the Navier-Stokes system 2.1.). Then

$$
\mathcal{H}^{1}(S)=0
$$

Remark 3.3. This particular statement has been chosen since its technical conditions are reasonably easy to read, but several variants of it could have been chosen. For instance, the above result is restricted to a bounded domain $D$, in order to use some facts already proved in the literature, but other domains and boundary conditions can certainly be treated. The conditions on the function $g$ are not optimal. We chose the $(1 / 2-\varepsilon)$-Hölder continuity in view of the results in the case of white noise forces, but a different balance of time and space regularity could be used in order to have the necessary properties of $z$.

As a simple corollary of Theorem 3.2 it is possible to show the following result.

Theorem 3.4. Let

$$
f \in L^{p}((0, T) \times D) \cap L^{2}(0, T ; H)
$$

for some $p>\frac{5}{2}$ and let $(u, P)$ be a martingale suitable weak solution of the NavierStokes system (2.1). Suppose furthermore that the Brownian motion $B_{t}$ has trajectories in the Hilbert space $D\left(A^{\frac{1}{4}+\delta}\right)$ for some $\delta>0$. Then for $\mathbf{P}$-a.e. $\omega \in \Omega$,

$$
\mathcal{H}^{1}(S)=0 \text {. }
$$

The main result in the framework of the statistical solutions is the following. Let $\mu$ be a stationary solution. We assume that the marginal of $\mu$ in the second component is concentrated on the space $C^{\frac{1}{2}-\varepsilon}\left([0, \infty) ; D\left(A^{\frac{1}{4}+\beta}\right)\right.$ for some $\beta>\varepsilon>0$.

Theorem 3.5. Let $\mu$ be a stationary solution as above. Then for every time $t \geq 0$ the set of singular points at time $t$ is empty for $\mu$-almost every trajectory.

In other words, if $S_{t}(u)$ denotes the set of all $x \in D$ such that $(t, x)$ is a singular point for the function $u$, then for all given $t \geq 0$, the set $S_{t}(u)$ is empty for $\mu$-almost all trajectories $u$.

From this theorem, a regularity result for almost any initial condition can be deduced. First we define a measure on the space $H$ of initial conditions given by a stationary solution $\mu$. Since weak solutions are continuous from $[0,+\infty)$ to $H$ with the weak topology, the map

$$
p_{0}:(u, B) \in \mathcal{S} \rightarrow u(0) \in H
$$

is well defined and measurable. Hence it is possible to consider the image measure of $\mu$ with respect to $p_{0}$. Denote by $\mu_{0}$ such a measure on $H$.

Corollary 3.6. For each $t \in(0,+\infty)$, for $\mu_{0}$-almost every initial condition $u_{0}$, there is a martingale suitable weak solution $u\left(\cdot, u_{0}\right)$, starting at $u_{0}$, such that

$$
S_{t}\left(u\left(\cdot, u_{0}\right)\right)=\emptyset \quad \mathbf{P} \text {-a.s. }
$$

The measure $\mathbf{P}$ in the last statement depends on the martingale solution $u\left(\cdot, u_{0}\right)$. An explicit form of $\mathbf{P}$ will be given in the proof of the corollary.

The two results given above on stationary solutions are true even if $B \equiv 0$ (see [9]). They are entirely due to the stationarity of the process $u$, and not to 
the presence of noise. Thus they have an intrinsic interest but do not answer the question of the effect of noise perturbations on the emergence of singularities.

Consider the case $B=0$. In principle it may happen that the only stationary solution is a Dirac measure $\delta_{u_{*}}$, where $u_{*}$ is a deterministic stationary solution. Then Theorem 3.5 and Corollary 3.6 are trivial, since regularity of deterministic stationary solutions is well known.

An effect of the noise perturbation is to avoid a situation like this.

Theorem 3.7. Assume that the Brownian motion $B$ is non-degenerate, in the sense of Section [6.1] below. Then the support of $\mu_{0}$ is the whole space $H$.

Remark 3.8. An analogue of Theorem 3.5 seems to hold in the case of space stationary (or space homogeneous, in the language of turbulence theory) solutions. Precisely, a slightly informal proof shows that a space-stationary solution (not necessarily stationary in time) has the property that for all given $x \in \mathbf{R}^{3}$, the set $S_{x}(u)$ is empty for $\mathbf{P}$-a.e. $\omega \in \Omega$, where $S_{x}(u)$ denotes the set of all $t \geq 0$ such that $(t, x)$ is a singular point for the function $u(\omega)$.

\section{Dimensionless estimates}

In this section and in the next it will be assumed, without loss of generality, that $0 \in D$, and we shall work on the time interval $(-1,1)$. The reason is that restricting the local arguments around $t=0$ and $x=0$ will simplify the notations. The ball of radius $r$ centred at the origin will be denoted by $B_{r} \subset \mathbf{R}^{3}$.

In this section we assume that

$$
f \in L^{p}((-1,1) \times D) \cap L^{2}(-1,1 ; H) \quad \text { and } \quad g \in C^{\frac{1}{2}-\varepsilon}\left([-1,1] ; D\left(A^{\beta}\right)\right)
$$

for some $p>2$ and $\beta>\varepsilon>0$, in such a way that the solution $(z, Q)$ of the Stokes system enjoys, among others, the following properties:

$$
z \in L^{\infty}\left(-1,1 ; L_{\mathrm{loc}}^{4}(D)\right)
$$

and

$$
\frac{1}{r} \iint_{Q_{r}^{*}}|\nabla z|^{2} \rightarrow 0, \quad r \rightarrow 0 .
$$

Remark 4.1. It would suffice to take $z \in L^{p}\left(-1,1 ; L_{\text {loc }}^{4}(D)\right)$ for a sufficiently large $p$ instead of $p=\infty$, but this generality has no influence on the final theorem, since stronger conditions on $z$, and so on $f$ and $g$, will be imposed in the next section.

In this section and in the next one, we will often use the following local version of Sobolev inequalities. If $u \in H^{1}\left(\mathbf{R}^{3}\right)$, then

$$
\int_{B_{r}}|u|^{q} \leq C\left(\int_{B_{r}}|\nabla u|^{2}\right)^{a}\left(\int_{B_{r}}|u|^{2}\right)^{\frac{q}{2}-a}+\frac{C}{r^{2 a}}\left(\int_{B_{r}}|u|^{2}\right)^{\frac{q}{2}},
$$

where $q \in[2,6]$ and $a=\frac{3}{4}(q-2)$.

Following Caffarelli, Kohn and Nirenberg [2], we introduce some dimensionless quantities (in the sense that they are invariant under the Leray scaling (1.1)). For every vector field $v$ and scalar field $\pi$ (definitions are not restricted to solutions to 
equation (2.4) $)$, we set

$$
\begin{aligned}
A_{*}(v, r) & =\sup _{-\frac{7}{8} r^{2} \leq t \leq \frac{1}{8} r^{2}} \frac{1}{r} \int_{B_{r}}|v(t)|^{2}, \\
\delta_{*}(v, r) & =\frac{1}{r} \iint_{Q_{r}^{*}}|\nabla v|^{2}, \\
G_{*}(v, r) & =\frac{1}{r^{2}} \iint_{Q_{r}^{*}}|v|^{3}, \\
K_{*}(\pi, r) & =\frac{1}{r^{\frac{13}{4}}} \int_{-\frac{7}{8} r^{2}}^{\frac{1}{8} r^{2}}\left(\int_{B_{r}}|\pi|\right)^{\frac{5}{4}} d t, \\
J_{*}(v, \pi, r) & =\frac{1}{r^{2}} \iint_{Q_{r}^{*}}|v| \cdot|\pi|, \\
H_{*}(v, r) & =\left.\frac{1}{r^{2}} \iint_{Q_{r}^{*}}|v| \cdot|| v\right|^{2}-\overline{|v|_{r}^{2} \mid,}
\end{aligned}
$$

where $\overline{|v|_{r}^{2}}$ is the average of $v$ on the ball $B_{r}$ and $Q_{r}^{*}$ is the space-time cylinder $\left(-\frac{7}{8} r^{2}, \frac{1}{8} r^{2}\right) \times B_{r}(0)$. The following new quantities will be used to estimate the new terms in (2.8) that were not present in the original local energy inequality in 2]:

$$
\begin{aligned}
\Gamma_{*}(v, r) & =\frac{1}{r^{\frac{4}{3}}} \int_{-\frac{7}{8} r^{2}}^{\frac{1}{8} r^{2}}\left(\int_{B_{r}}|v|^{4}\right)^{\frac{2}{3}} d t, \\
Z_{*}(v, r) & =\frac{1}{r} \iint_{Q_{r}^{*}}|v|^{4}, \\
\Theta_{*}(v, r) & =\int_{-\frac{7}{8} r^{2}}^{\frac{1}{8} r^{2}}\left(\int_{B_{r}}|v|^{4}\right)^{2} d t .
\end{aligned}
$$

We will omit $v$ and $\pi$ in the notations $A_{*}(v, r), K_{*}(\pi, r), \ldots$ when they are the solutions to equation (2.4) (thus, in such a case, we will simply write $A_{*}(r), K_{*}(r)$, $J_{*}(r)$, for instance). The main result of this section is

Theorem 4.2. Assume (4.1) and let $(z, Q)$ be the solution of the Stokes system. Let $(u, P)$ be a suitable weak solution of the Navier-Stokes equations. Then, given $\varepsilon_{1}>0$, there exists $\varepsilon_{2}>0$ (depending on the norm of $z$ in $L^{\infty}\left(-1,1 ; L^{4}\left(B_{1}\right)\right.$ ) but not on $u$ and $P$ ) such that

$$
\left.\limsup _{r \rightarrow 0} \delta_{*}(v, r) \leq \varepsilon_{2} \quad \text { (or equivalently } \limsup _{r \rightarrow 0} \delta_{*}(u, r) \leq \varepsilon_{2}\right)
$$

implies

$$
\limsup _{r \rightarrow 0}\left(A_{*}(v, r)+G_{*}(v, r)+\Gamma_{*}(v, r)+H_{*}(v, r)+J_{*}(r)+K_{*}(r)\right) \leq \varepsilon_{1}
$$

and

$$
\limsup _{r \rightarrow 0}\left(A_{*}(u, r)+G_{*}(u, r)+\Gamma_{*}(u, r)+H_{*}(u, r)\right) \leq \varepsilon_{1},
$$

where $(v, \pi)$ is the solution of the modified equation, as obtained in (2.2).

The proof is based on a sequence of preliminary estimates. The following one is proved by using the local energy inequality. The analysis of singularities (or blow-up) of solutions corresponds to the analysis of the concentration and transfer 
of energy to small scales. The energy inequality, properly localised by means of suitable test functions, allows us to control the energy in a ball $B_{\frac{r}{2}}$ by other quantities (many of them having a physical meaning, see Gallavotti [10]) on the larger ball $B_{r}$. Roughly speaking, these quantities are further estimated by the energy in a larger ball $B_{\rho}$. The final result will be that, if some of these quantities are sufficiently small in some ball, then all the quantities are controlled in smaller balls, ultimately preventing the blow-up.

Lemma 4.3. Let $(v, \pi)$ be as in the previous theorem and let $r$ be given. Then (up to an absolute constant $C$ that is omitted for simplicity of notation)

$$
\begin{aligned}
A_{*}\left(\frac{r}{2}\right)+2 \delta_{*}\left(\frac{r}{2}\right) \leq & G_{*}(r)^{\frac{2}{3}}+H_{*}(r)+G_{*}(r)^{\frac{2}{3}} G_{*}(z, r)^{\frac{1}{3}}+G_{*}(r)^{\frac{1}{3}} G_{*}(z, r)^{\frac{2}{3}} \\
& +\delta_{*}(r)^{\frac{1}{2}} \Gamma_{*}(r)^{\frac{3}{8}} \Theta_{*}(z, r)^{\frac{1}{8}}+\delta_{*}(r)^{\frac{1}{2}} Z_{*}(z, r)^{\frac{1}{2}}+J_{*}(r) .
\end{aligned}
$$

Proof. Let $r<\rho$ be given. A general estimate between the balls $B_{r}$ and $B_{\rho}$ is proved, and at the end it is applied to the balls $B_{\frac{r}{2}}$ and $B_{r}$. Consider the function $\varphi$ having the following properties (in this proof, $C$ will denote a generic positive constant independent of $v, \pi, z, f$ and $r, \rho)$ :

$$
\begin{aligned}
0 \leq \varphi & \leq 1, \quad \varphi=0 \text { outside } Q_{\rho}^{*}, \quad \varphi=1 \text { in } Q_{r}^{*} \\
|\nabla \varphi| & \leq \frac{C}{\rho-r}, \quad\left|\frac{\partial \varphi}{\partial t}\right|+|\triangle \varphi| \leq \frac{C}{(\rho-r)^{2}}
\end{aligned}
$$

and use it as a test function in the local energy inequality (2.8). In this way the following Caccioppoli type estimate is obtained:

$$
\begin{aligned}
\int_{B_{r}}|v(t)|^{2}+2 \iint_{Q_{r}^{*}}|\nabla v|^{2} \leq & \frac{C}{(\rho-r)^{2}} \iint_{Q_{\rho}^{*}}|v|^{2}+\iint_{Q_{\rho}^{*}}|v|^{2}(v \cdot \nabla) \varphi \\
& +\frac{C}{\rho-r} \iint_{Q_{\rho}^{*}}|v|^{2}|z|+\frac{C}{\rho-r} \iint_{Q_{\rho}^{*}} 2|v||z|^{2} \\
& +\iint_{Q_{\rho}^{*}}|z||v||\nabla v|+\iint_{Q_{\rho}^{*}}|z|^{2}|\nabla v| \\
& +\frac{C}{\rho-r} \iint_{Q_{\rho}^{*}} 2|v||\pi| .
\end{aligned}
$$

The term $\iint|v|^{2}(v \cdot \nabla) \varphi$ requires special care; otherwise it produces a term of third order in $v$ which does not allow us to close the inequality . Using the incompressibility condition, it follows that (here the geometric structure of the nonlinear term of the Navier-Stokes equation plays an essential role, the same computations cannot be repeated for a generic quadratic nonlinearity)

$$
\iint_{Q_{\rho}^{*}}|v|^{2}(v \cdot \nabla) \varphi=\iint_{Q_{\rho}^{*}}\left(|v|^{2}-\overline{|v|_{\rho}^{2}}\right)(v \cdot \nabla) \varphi .
$$


Therefore the previous inequality implies that

$$
\begin{aligned}
& \int_{B_{r}}|v(t)|^{2}+2 \iint_{Q_{r}^{*}}|\nabla v|^{2} \\
& \quad \leq \frac{C}{(\rho-r)^{2}} \rho^{\frac{5}{3}}\left(\iint_{Q_{\rho}^{*}}|v|^{3}\right)^{\frac{2}{3}}+\left.\frac{C}{\rho-r} \iint_{Q_{\rho}^{*}}|| v\right|^{2}-\overline{|v|_{\rho}^{2} \mid}|v| \\
& \quad+\frac{C}{\rho-r}\left(\iint_{Q_{\rho}^{*}}|v|^{3}\right)^{\frac{2}{3}}\left(\iint_{Q_{\rho}^{*}}|z|^{3}\right)^{\frac{1}{3}}+\frac{C}{\rho-r}\left(\iint_{Q_{\rho}^{*}}|v|^{3}\right)^{\frac{1}{3}}\left(\iint_{Q_{\rho}^{*}}|z|^{3}\right)^{\frac{2}{3}} \\
& \quad+\left(\iint_{Q_{\rho}^{*}}|\nabla v|^{2}\right)^{\frac{1}{2}}\left(\int_{-\frac{7}{8} \rho^{2}}^{\frac{1}{8} \rho^{2}}\left(\int_{B_{\rho}}|v|^{4}\right)^{\frac{2}{3}} d t\right)^{\frac{3}{8}}\left(\int_{-\frac{7}{8} \rho^{2}}^{\frac{1}{8} \rho^{2}}\left(\int_{B_{\rho}}|z|^{4}\right)^{2} d t\right)^{\frac{1}{8}} \\
& \quad+\left(\iint_{Q_{\rho}^{*}}|\nabla v|^{2}\right)^{\frac{1}{2}}\left(\iint_{Q_{\rho}^{*}}|z|^{4}\right)^{\frac{1}{2}}+\frac{C}{\rho-r} \iint_{Q_{\rho}^{*}} 2|v||\pi|,
\end{aligned}
$$

since

$$
\begin{aligned}
\iint_{Q_{\rho}^{*}}|z||v||\nabla v| \leq\left(\iint_{Q_{\rho}^{*}}|\nabla v|^{2}\right)^{\frac{1}{2}}\left(\iint_{Q_{\rho}^{*}}|z|^{2}|v|^{2}\right)^{\frac{1}{2}} \\
\quad \leq\left(\iint_{Q_{\rho}^{*}}|\nabla v|^{2}\right)^{\frac{1}{2}}\left(\int_{-\frac{7}{8} \rho^{2}}^{\frac{1}{8} \rho^{2}}\left(\int_{B_{\rho}}|v|^{4}\right)^{\frac{1}{2}}\left(\int_{B_{\rho}}|z|^{4}\right)^{\frac{1}{2}} d t\right)^{\frac{1}{2}} \\
\quad \leq\left(\iint_{Q_{\rho}^{*}}|\nabla v|^{2}\right)^{\frac{1}{2}}\left(\int_{-\frac{7}{8} \rho^{2}}^{\frac{1}{8} \rho^{2}}\left(\int_{B_{\rho}}|v|^{4}\right)^{\frac{2}{3}} d t\right)^{\frac{3}{8}}\left(\int_{-\frac{7}{8} \rho^{2}}^{\frac{1}{8} \rho^{2}}\left(\int_{B_{\rho}}|z|^{4}\right)^{2} d t\right)^{\frac{1}{8}} .
\end{aligned}
$$

Therefore

$$
\begin{aligned}
A_{*}(r)+2 \delta_{*}(r) \leq & \frac{C}{r(\rho-r)^{2}} \rho^{\frac{5}{3}}\left(\rho^{2} G_{*}(\rho)\right)^{\frac{2}{3}}+\frac{C}{r(\rho-r)}\left(\rho^{2} H_{*}(\rho)\right) \\
& +\frac{C}{r(\rho-r)}\left(\rho^{2} G_{*}(\rho)\right)^{\frac{2}{3}}\left(\rho^{2} G_{*}(z, \rho)\right)^{\frac{1}{3}} \\
& +\frac{C}{r(\rho-r)}\left(\rho^{2} G_{*}(\rho)\right)^{\frac{1}{3}}\left(\rho^{2} G_{*}(z, \rho)\right)^{\frac{2}{3}} \\
& +r^{-1}\left(\rho \delta_{*}(\rho)\right)^{\frac{1}{2}}\left(\rho^{\frac{4}{3}} \Gamma_{*}(\rho)\right)^{\frac{3}{8}}\left(\Theta_{*}(z, \rho)\right)^{\frac{1}{8}} \\
& +r^{-1}\left(\rho \delta_{*}(\rho)\right)^{\frac{1}{2}}\left(\rho Z_{*}(z, \rho)\right)^{\frac{1}{2}}+\frac{C}{r(\rho-r)}\left(\rho^{2} J_{*}(v, \pi, \rho)\right) \\
\leq & \frac{C \rho^{3}}{r(\rho-r)^{2}} G_{*}(\rho)^{\frac{2}{3}}+\frac{C \rho^{2}}{r(\rho-r)} H_{*}(\rho) \\
& +\frac{C \rho^{2}}{r(\rho-r)} G_{*}(\rho)^{\frac{2}{3}} G_{*}(z, \rho)^{\frac{1}{3}}+\frac{C \rho^{2}}{r(\rho-r)} G_{*}(\rho)^{\frac{1}{3}} G_{*}(z, \rho)^{\frac{2}{3}} \\
& +\rho r^{-1} \delta_{*}(\rho)^{\frac{1}{2}} \Gamma_{*}(\rho)^{\frac{3}{8}} \Theta_{*}(z, \rho)^{\frac{1}{8}}+\rho r^{-1} \delta_{*}(\rho)^{\frac{1}{2}} Z_{*}(z, \rho)^{\frac{1}{2}} \\
& +\frac{C \rho^{2}}{r(\rho-r)} J_{*}(v, \pi, \rho) .
\end{aligned}
$$

This yields the inequality of the lemma. 
In the sequel, $C_{z}$ will be a generic positive constant depending only on the norm of $z$ in $L^{\infty}\left(-1,1 ; L^{4}\left(B_{1}\right)\right)$ but not on $u, \pi, v, z$ itself, or $r$. Then

$$
G_{*}(z, r) \leq C_{z} r^{\frac{3}{4}}, \quad \Theta_{*}(z, r) \leq C_{z}, \quad Z_{*}(z, r) \leq C_{z} r .
$$

Moreover, the following inequalities, mostly proved in Caffarelli, Kohn and Nirenberg [2], hold.

Lemma 4.4. For any divergence-free vector field $v$ and for all $r$ ( $v$ is omitted)

$$
\begin{aligned}
H_{*}(r) & \leq C\left[G_{*}(r)^{\frac{2}{3}}+A_{*}(r) \delta_{*}(r)\right], \\
G_{*}(r) & \leq C\left[A_{*}(r)^{\frac{3}{2}}+A_{*}(r)^{\frac{3}{4}} \delta_{*}(r)^{\frac{3}{4}}\right], \\
\Gamma_{*}(r) & \leq C\left[A_{*}(r)^{\frac{4}{3}}+A_{*}(r)^{\frac{1}{3}} \delta_{*}(r)\right] .
\end{aligned}
$$

Proof. The first two inequalities are proved in Caffarelli, Kohn and Nirenberg [2]. For the third one, by the Sobolev inequality,

$$
\int_{B_{r}}|v|^{4} \leq\left(\int_{B_{r}}|\nabla v|^{2}\right)^{\frac{3}{2}}\left(\int_{B_{r}}|v|^{2}\right)^{\frac{1}{2}}+\frac{C}{r^{3}}\left(\int_{B_{r}}|v|^{2}\right)^{2} ;
$$

hence

$$
\begin{aligned}
\int_{-\frac{7}{8} r^{2}}^{\frac{1}{8} r^{2}}\left(\int_{B_{r}}|v|^{4}\right)^{\frac{2}{3}} d t \leq & \left(\sup _{-\frac{7}{8} r^{2} \leq t \leq \frac{1}{8} r^{2}} \int_{B_{r}}|v(t)|^{2}\right)^{\frac{1}{3}} \iint_{Q_{\rho}^{*}}|\nabla v|^{2} \\
& +C\left(\sup _{-\frac{7}{8} r^{2} \leq t \leq \frac{1}{8} r^{2}} \int_{B_{r}}|v(t)|^{2}\right)^{\frac{4}{3}} .
\end{aligned}
$$

This implies the third inequality.

The following corollary gives a simplified version of Lemma 4.3

Corollary 4.5. Let $(v, \pi)$ be as in Theorem 4.2 and let $r>0$ be given. Then, for a suitable $\alpha>0$ and for all $r \leq 1$ such that also $\delta_{*}(r) \leq 1$ (C is an absolute constant),

$$
\begin{aligned}
A_{*}\left(\frac{r}{2}\right)+2 \delta_{*}\left(\frac{r}{2}\right) \leq & C\left[G_{*}(r)^{\frac{2}{3}}+J_{*}(r)\right]+C_{z} A_{*}(r)\left[r^{\alpha}+\delta_{*}(r)^{\alpha}\right] \\
& +C_{z}\left[r^{\frac{1}{2}}+\delta_{*}(r)^{\frac{1}{2}}\right] .
\end{aligned}
$$

Proof. The use of the various inequalities proved above in the previous lemma gives

$$
\begin{aligned}
A_{*}\left(\frac{r}{2}\right)+2 \delta_{*}\left(\frac{r}{2}\right) \leq & C G_{*}(r)^{\frac{2}{3}}+C\left[G_{*}(r)^{\frac{2}{3}}+A_{*}(r) \delta_{*}(r)\right] \\
& +C C_{z} r^{\frac{1}{4}}\left[A_{*}(r)^{\frac{3}{2}}+A_{*}(r)^{\frac{3}{4}} \delta_{*}(r)^{\frac{3}{4}}\right]^{\frac{2}{3}} \\
& +C C_{z} r^{\frac{1}{2}}\left[A_{*}(r)^{\frac{3}{2}}+A_{*}(r)^{\frac{3}{4}} \delta_{*}(r)^{\frac{3}{4}}\right]^{\frac{1}{3}} \\
& +C C_{z} \delta_{*}(r)^{\frac{1}{2}}\left[A_{*}(r)^{\frac{4}{3}}+A_{*}(r)^{\frac{1}{3}} \delta_{*}(r)\right]^{\frac{3}{8}} \\
& +C_{z} r^{\frac{1}{2}} \delta_{*}(r)^{\frac{1}{2}}+C J_{*}(r) .
\end{aligned}
$$

Inspection of the various terms reveals that it is sufficient to apply Young inequality several times (always in order to raise the terms $A_{*}(r)^{\beta}$ to the power one) to prove 
the statement of the lemma. The power $\alpha$ will be the smaller of the powers of $r, \delta_{*}(r)$, which dominates the other powers since it is assumed that $r \leq 1$ and $\delta_{*}(r) \leq 1$.

If the two terms $G_{*}(r)^{\frac{2}{3}}$ and $J_{*}(r)$ are neglected in the previous inequality, this can be used, under the assumptions of the theorem, to prove the smallness of $A_{*}(r)$. In order to treat the remaining two terms, the following basic estimates proved in Caffarelli, Kohn and Nirenberg [2] are needed. Since the relation between the pressure $\pi$ and $v$ is not the same as in [2], the estimates for $J_{*}(\rho)$ and $K_{*}(\rho)$ are slightly different. Thus we repeat their statement for the reader's convenience.

Lemma 4.6. For all $r \leq \rho$

$$
G_{*}(r) \leq C\left[\left(\frac{r}{\rho}\right)^{3} A_{*}(\rho)^{\frac{3}{2}}+\left(\frac{\rho}{r}\right)^{3} A_{*}(\rho)^{\frac{3}{4}} \delta_{*}(\rho)^{\frac{3}{4}}\right],
$$

and for all $2 r \leq \rho$,

$$
\begin{aligned}
J_{*}(r) & \leq C\left[\left(\frac{r}{\rho}\right)^{\frac{1}{5}} A_{*}(\rho)^{\frac{1}{5}} G_{*}(\rho)^{\frac{1}{5}} K_{*}(\rho)^{\frac{4}{5}}+\left(\frac{\rho}{r}\right)^{2} A_{*}(\rho)^{\frac{1}{2}} \delta_{*}(v+z, \rho)\right], \\
K_{*}(r) & \leq C\left[\left(\frac{r}{\rho}\right)^{\frac{1}{2}} K_{*}(\rho)+\left(\frac{\rho}{r}\right)^{\frac{5}{4}} A_{*}(v+z, \rho)^{\frac{5}{8}} \delta_{*}(v+z, \rho)^{\frac{5}{8}}\right] .
\end{aligned}
$$

Finally, the proof of Theorem 4.2 follows.

Proof. From the inequalities of the two lemmas it is clearly sufficient to prove the result for $A_{*}(r)$. However, $A_{*}(r)$ cannot be controlled explicitly in terms of quantities which are small by assumption, since $K_{*}(r)$ is also recursively estimated by itself. Therefore it is necessary to control $A_{*}(r)$ and $K_{*}(r)$ simultaneously.

If we choose $\varepsilon_{2} \leq 1$ and $r$ sufficiently small, the assumptions of the corollary are satisfied. Such a choice of $\varepsilon_{2}$ and $r$ (or $\rho$ ) is tacitly assumed.

From the previous lemma and corollary, if $2 r \leq \rho$,

$$
\begin{aligned}
A_{*}\left(\frac{r}{2}\right) \leq & C\left(\frac{r}{\rho}\right)^{2} A_{*}(\rho)+C\left(\frac{\rho}{r}\right)^{2} A_{*}(\rho)^{\frac{1}{2}} \delta_{*}(\rho)^{\frac{1}{2}}+C\left(\frac{\rho}{r}\right)^{2} A_{*}(\rho)^{\frac{1}{2}} \delta_{*}(v+z, \rho) \\
& +C\left(\frac{r}{\rho}\right)^{\frac{1}{5}}\left[A_{*}(\rho)^{\frac{1}{2}}+A_{*}(\rho)^{\frac{7}{20}} \delta_{*}(\rho)^{\frac{7}{20}}\right] K_{*}(\rho)^{\frac{4}{5}} \\
& +C_{z} A_{*}(r)\left(r^{\alpha}+\delta_{*}(r)^{\alpha}\right)+C_{z}\left(r^{\frac{1}{2}}+\delta_{*}(r)^{\frac{1}{2}}\right)
\end{aligned}
$$

and

$$
K_{*}\left(\frac{r}{2}\right) \leq C\left(\frac{r}{\rho}\right)^{\frac{1}{2}} K_{*}(\rho)+C\left(\frac{\rho}{r}\right)^{\frac{5}{4}} A_{*}(v+z, \rho)^{\frac{5}{8}} \delta_{*}(v+z, \rho)^{\frac{5}{8}}
$$

If the ratio $\frac{r}{\rho} \leq \frac{1}{2}$ is chosen in such a way that ( $C$ is the previous constant)

$$
C\left(\frac{r}{\rho}\right)^{\frac{1}{5}} \leq \frac{1}{8}
$$


it follows that

$$
\begin{aligned}
A_{*}\left(\frac{r}{2}\right) \leq & \frac{1}{8} A_{*}(\rho)+C A_{*}(\rho)^{\frac{1}{2}} \delta_{*}(\rho)^{\frac{1}{2}}+\frac{1}{8}\left[A_{*}(\rho)^{\frac{1}{2}}+A_{*}(\rho)^{\frac{7}{20}} \delta_{*}(\rho)^{\frac{7}{20}}\right] K_{*}(\rho)^{\frac{4}{5}} \\
& +C A_{*}(\rho)^{\frac{1}{2}} \delta_{*}(v+z, \rho)+C_{z} A_{*}(\rho)\left[\rho^{\alpha}+\delta_{*}(\rho)^{\alpha}\right]+C_{z}^{\frac{1}{2}}\left[\rho^{\frac{1}{2}}+\delta_{*}(\rho)^{\frac{1}{2}}\right] \\
\leq & \frac{1}{8} A_{*}(\rho)+\frac{1}{8}\left[A_{*}(\rho)^{\frac{1}{2}}+A_{*}(\rho)^{\frac{7}{20}} \delta_{*}(\rho)^{\frac{7}{20}}\right] K_{*}(\rho)^{\frac{4}{5}} \\
& +C_{z} A_{*}(\rho)\left[\rho^{\alpha}+\delta_{*}(\rho)^{\alpha}+\delta_{*}(z, \rho)^{\alpha}\right]+C_{z}^{\frac{1}{2}}\left[\rho^{\frac{1}{2}}+\delta_{*}(\rho)^{\frac{1}{2}}\right]
\end{aligned}
$$

(having applied again the Young inequality, with a new choice of $\alpha$ if necessary), and

$$
\begin{aligned}
K_{*}\left(\frac{r}{2}\right) & \leq \frac{1}{8} K_{*}(\rho)+C A_{*}(v+z, \rho)^{\frac{5}{8}} \delta_{*}(v+z, \rho)^{\frac{5}{8}} \\
& \leq \frac{1}{8} K_{*}(\rho)+C A_{*}(\rho)^{\frac{5}{8}} \delta_{*}(v+z, \rho)^{\frac{5}{8}}+C A_{*}(z, \rho)^{\frac{5}{8}} \delta_{*}(v+z, \rho)^{\frac{5}{8}}
\end{aligned}
$$

Now $\varepsilon_{2}$ and $\rho_{0}$ are chosen in such a way that for all $\rho \leq \rho_{0}$,

$$
\begin{aligned}
C_{z}\left[\rho^{\alpha}+\delta_{*}(\rho)^{\alpha}+\delta_{*}(z, \rho)^{\alpha}\right] & \leq \frac{1}{8} \\
C_{z}\left[r^{\frac{1}{2}}+\delta_{*}(r)^{\frac{1}{2}}\right] & \leq \frac{\varepsilon_{1}}{8} \\
C\left(\frac{\rho}{r}\right)^{\frac{5}{4}} \delta_{*}(v+z, \rho)^{\frac{5}{8}} & \leq \frac{1}{8} \\
C A_{*}(z, \rho)^{\frac{5}{8}} \delta_{*}(v+z, \rho)^{\frac{5}{8}} & \leq\left(\frac{\varepsilon_{1}}{8}\right)^{\frac{5}{8}}, \\
\delta_{*}(\rho)^{\frac{7}{20}} & \leq \varepsilon_{1} .
\end{aligned}
$$

Therefore,

$$
\begin{aligned}
& A_{*}\left(\frac{r}{2}\right) \leq \frac{1}{4} A_{*}(\rho)+\frac{1}{8}\left[A_{*}(\rho)^{\frac{1}{2}}+A_{*}(\rho)^{\frac{7}{20}} \delta_{*}(\rho)^{\frac{7}{20}}\right] K_{*}(\rho)^{\frac{4}{5}}+\frac{\varepsilon_{1}}{8}, \\
& K_{*}\left(\frac{r}{2}\right) \leq \frac{1}{8} K_{*}(\rho)+\frac{1}{8} A_{*}(\rho)^{\frac{5}{8}}+\left(\frac{\varepsilon_{1}}{8}\right)^{\frac{5}{8}} .
\end{aligned}
$$

Now, let $L$ be the maximum between $A_{*}\left(\rho_{0}\right)$ and $\left(K_{*}\left(\rho_{0}\right)\right)^{\frac{8}{5}}$, and $\gamma$ the number such that $\frac{r}{2}=\gamma \rho$, when $r$ and $\rho$ are related by the ratio chosen above. Then

$$
A_{*}\left(\gamma \rho_{0}\right) \leq \frac{1}{4} L+\frac{1}{8}\left[L^{\frac{1}{2}}+L^{\frac{7}{20}} \delta_{*}\left(\rho_{0}\right)^{\frac{7}{20}}\right] L^{\frac{1}{2}}+\frac{\varepsilon_{1}}{8} \leq \frac{1}{2} L+\frac{\varepsilon_{1}}{4}
$$

(since

$$
\frac{1}{8}\left(L^{\frac{1}{2}}+L^{\frac{7}{20}} \delta_{*}\left(\rho_{0}\right)^{\frac{7}{20}}\right) L^{\frac{1}{2}} \leq \frac{1}{4} L
$$

if $L \geq 1$, using also $\delta_{*}\left(\rho_{0}\right) \leq 1$, while

$$
\frac{1}{8}\left(L^{\frac{1}{2}}+L^{\frac{7}{20}} \delta_{*}\left(\rho_{0}\right)^{\frac{7}{20}}\right) L^{\frac{1}{2}} \leq \frac{1}{8} L+\frac{1}{8} \delta_{*}\left(\rho_{0}\right)^{\frac{7}{20}} \leq \frac{\varepsilon_{1}}{8}
$$

if $L<1$, using also $\left.\delta_{*}\left(\rho_{0}\right) \leq \varepsilon_{1}\right)$, and

$$
K_{*}\left(\gamma \rho_{0}\right) \leq \frac{1}{4} L^{\frac{5}{8}}+\left(\frac{\varepsilon_{1}}{8}\right)^{\frac{5}{8}}
$$

Iteratively this procedure yields the result of the theorem. 


\section{LOCAL REGULARITY}

In this section, following Caffarelli, Kohn and Nirenberg [2], we will prove that the point $(0,0)$ is regular if $\lim \sup _{r \rightarrow 0} \delta_{*}(r)$ is sufficiently small. From this result we shall deduce (as in [2]) that the set of singular points has 1-dimensional Hausdorff measure equal to zero, since the same property holds true for the set of points where $\lim \sup _{r \rightarrow 0} \delta_{*}(r)$ is not small.

We assume that (4.1) holds, in such a way that the solution $z$ of the Stokes problem satisfies the condition

$$
z \in L^{\infty}\left(-1,1 ; L_{\mathrm{loc}}^{q}(D)\right) \quad \text { for } q>6 .
$$

Following Gallavotti [10], the quantities

$$
\begin{aligned}
& a(r)=\sup _{-r^{2} \leq t \leq 0} \frac{1}{r^{3}} \int_{B_{r}}|v(t)|^{2}, \\
& d(r)=\frac{1}{r^{3}} \iint_{Q_{r}}|\nabla v|^{2}, \\
& g(r)=\frac{1}{r^{5}} \iint_{Q_{r}}|v|^{3}, \\
& s(r)=\frac{1}{r^{5}} \iint_{Q_{r}}|z|^{6}, \\
& z(r)=\frac{1}{r^{5}} \iint_{Q_{r}}|z|^{4}, \\
& l(r)=\frac{1}{r^{5}} \iint_{Q_{r}}|v| \cdot\left|\pi-\overline{\pi_{r}}\right|, \\
& k(r)=\frac{1}{r^{\frac{23}{4}}} \int_{-r^{2}}^{0}\left(\int_{B_{r}}|\pi|\right)^{\frac{5}{4}} d t
\end{aligned}
$$

can be introduced, where $\overline{\pi_{r}}$ is the average of $\pi$ on the ball $B_{r}$ and $Q_{r}$ is the space-time cylinder $\left(-r^{2}, 0\right) \times B_{r}(0)$.

The following basic estimates are mostly proved in $[2]$.

Lemma 5.1. For all $r>0$

$$
g(r) \leq C a(r)^{\frac{3}{4}}\left[a(r)^{\frac{3}{4}}+d(r)^{\frac{3}{4}}\right],
$$

and, if $4 r \leq \rho$,

$$
\begin{aligned}
l(r) \leq & C g(r)^{1 / 3}\left[\left(\frac{r}{\rho}\right)^{5 / 3} g(v+z, \rho)^{2 / 3}+g(v+z, 2 r)^{2 / 3}\right] \\
& +C r g(r)^{1 / 3} \sup _{-r^{2}<t<0} \int_{2 r<y<\rho} \frac{|v+z|^{2}}{|y|^{4}} d y \\
& +C r^{-2}\left(\frac{r}{\rho}\right)^{7 / 5} a(r)^{1 / 5} g(r)^{1 / 5} k(\rho)^{4 / 5} .
\end{aligned}
$$

Proof. Lemma 3.1 and Lemma 3.2 of Caffarelli, Kohn and Nirenberg [2] are used with minor changes (the contribution given by the term $|v|$ has to be treated separately from that of $|v+z|$ ), and the desired property is obtained by passing from dimensionless quantities to the new quantities $a, g, k, l$ and $d$. 
The first result proceeds as follows. Condition (5.3) is not required in Caffarelli, Kohn and Nirenberg [2]. On the other hand, this assumption is not restrictive in view of the final result.

Proposition 5.2. There exist three constants $\varepsilon_{1}, \varepsilon_{2}, \varepsilon_{3}>0$ such that, if $(v, \pi)$ satisfies

$$
\begin{gathered}
\iint_{Q_{1}}\left(|v|^{3}+|v| \cdot|\pi|\right) d x d t+\int_{-1}^{0}\left(\int_{B_{1}}|\pi| d x\right)^{5 / 4} d t \leq \varepsilon_{1}, \\
\qquad \int_{Q_{1}}|\nabla v|^{2} d x d t \leq \varepsilon_{2}, \\
\sup _{-1<t<0} \int_{B_{1}}|z|^{q} d x \leq \varepsilon_{3}, \quad q>6,
\end{gathered}
$$

then $(0,0)$ is a regular point for $v$.

Proof. Let $(a, s) \in Q_{1 / 2}(0,0)$; then $Q_{1 / 2}(a, s) \subset Q_{1}(0,0)$. In the rest of this proof, if not explicitly specified, balls and cylinders are centred at the point $(a, s)$.

For every $n$, set $r_{n}=2^{-n}$. Apply the local energy inequality with

$$
\varphi_{n}(t, x)=\chi(t, x) \psi_{n}(t-s, x-a),
$$

where $\chi$ is a smooth function with compact support in $Q_{\frac{1}{3}}$ equal to 1 in $Q_{\frac{1}{4}}$, and

$$
\psi_{n}(t, x)=\frac{1}{\left(r_{n}^{2}-t\right)^{3 / 2}} \mathrm{e}^{-\frac{|x|^{2}}{4\left(r_{n}^{2}-t\right)}}
$$

Since $\varphi_{n} \geq 0$,

$$
\begin{aligned}
\partial_{t} \varphi_{n}+\Delta \varphi_{n} & =0 \text { on } Q_{1 / 4}, & \left|\partial_{t} \varphi_{n}+\Delta \varphi_{n}\right| & \leq C \text { everywhere, } \\
\frac{1}{C r_{n}^{3}} \leq \varphi_{n} & \leq C r_{n}^{3}, & \left|\nabla \varphi_{n}\right| & \leq \frac{C}{r_{y}^{4}} \quad \text { on } Q_{r_{n}}, \\
\varphi_{n} & \leq \frac{C}{r_{k}^{3}}, & \left|\nabla \varphi_{n}\right| & \leq \frac{C}{r_{k}^{4}} \text { on } Q_{r_{k-1}} \backslash Q_{r_{k}}, k \leq n,
\end{aligned}
$$

the local energy inequality, applied to the function $\varphi_{n}$, gives

$$
\begin{aligned}
\frac{1}{r_{n}^{3}} \int_{B_{r_{n}}}|v|^{2}+\frac{2}{r_{n}^{3}} \iint_{Q_{r_{n}}}|\nabla v|^{2} \\
\leq C \iint_{Q_{1}(0,0)}|v|^{2}+\iint_{Q_{\frac{1}{2}}}\left(|v|^{2}-2 v \cdot z\right)((v+z) \cdot \nabla) \varphi_{n} \\
\quad-\iint_{Q_{\frac{1}{2}}} \varphi_{n} z \cdot((v+z) \cdot \nabla) v-\iint_{Q_{\frac{1}{2}}} 2 \pi v \cdot \nabla \varphi_{n} \\
\leq C \iint_{Q_{1}(0,0)}|v|^{2}+\left|\iint_{Q_{\frac{1}{2}}} 2 \pi v \cdot \nabla \varphi_{n}\right| \\
\quad+\sum_{k=1}^{n} \frac{C}{r_{k}^{4}} \iint_{Q_{r_{k}}}\left(|v|^{3}+3|v|^{2}|z|+2|z|^{2}|v|\right) \\
\quad+\sum_{k=1}^{n} \frac{C}{r_{k}^{3}} \iint_{Q_{r_{k}}}\left(|v| \cdot|z| \cdot|\nabla v|+|z|^{2}|\nabla v|\right)
\end{aligned}
$$


We estimate all the terms in the second hand side as follows:

$$
\begin{aligned}
\sum_{k=1}^{n} \frac{C}{r_{k}^{4}} \iint_{Q_{r_{k}}}|v|^{3} & =\sum_{k=1}^{n} C r_{k} g\left(r_{k}\right) \\
\sum_{k=1}^{n} \frac{C}{r_{k}^{4}} \iint_{Q_{r_{k}}}|v|^{2}|z| & \leq \sum_{k=1}^{n} \frac{C}{r_{k}^{4}}\left(\iint_{Q_{r_{k}}}|v|^{3}\right)^{\frac{2}{3}}\left(\iint_{Q_{r_{k}}}|z|^{3}\right)^{\frac{1}{3}} \\
& \leq \sum_{k=1}^{n} C r_{k} g\left(r_{k}\right)^{\frac{2}{3}} g\left(z, r_{k}\right)^{\frac{1}{3}} \\
\sum_{k=1}^{n} \frac{C}{r_{k}^{4}} \iint_{Q_{r_{k}}}|v||z|^{2} & \leq \sum_{k=1}^{n} C r_{k} g\left(r_{k}\right)^{\frac{1}{3}} g\left(z, r_{k}\right)^{\frac{2}{3}} .
\end{aligned}
$$

Now the terms with the gradient part are estimated:

$$
\begin{aligned}
\iint_{Q_{r_{k}}}|\nabla v| \cdot|v| \cdot|z| & \leq\left(\iint_{Q_{r_{k}}}|\nabla v|^{2}\right)^{\frac{1}{2}}\left(\iint_{Q_{r_{k}}}|v|^{3}\right)^{\frac{1}{3}}\left(\iint_{Q_{r_{k}}}|z|^{6}\right)^{\frac{1}{6}} \\
& \leq r_{k}^{4} d\left(r_{k}\right)^{\frac{1}{2}} g\left(r_{k}\right)^{\frac{1}{3}} s\left(r_{k}\right)^{\frac{1}{6}}
\end{aligned}
$$

and

$$
\iint_{Q_{r_{k}}}|\nabla v| \cdot|z|^{2} \leq\left(\iint_{Q_{r_{k}}}|\nabla v|^{2}\right)^{\frac{1}{2}}\left(\iint_{Q_{r_{k}}}|z|^{4}\right)^{\frac{1}{2}} \leq r_{k}^{4} d\left(r_{k}\right)^{\frac{1}{2}} z\left(r_{k}\right)^{\frac{1}{2}},
$$

so at last

$$
C \sum_{k=1}^{n} \frac{1}{r_{k}^{3}} \iint_{Q_{r_{k}}}|\nabla v|\left(|z|^{2}+|z| \cdot|v|\right) \leq C \sum_{k=1}^{n} r_{k} d\left(r_{k}\right)^{\frac{1}{2}}\left[g\left(r_{k}\right)^{\frac{1}{3}} s\left(r_{k}\right)^{\frac{1}{6}}+z\left(r_{k}\right)^{\frac{1}{2}}\right] .
$$

The pressure term can be estimated as in Caffarelli, Kohn and Nirenberg [2]. In fact,

$$
\begin{aligned}
\left|\iint_{Q_{\frac{1}{2}}} 2 \pi v \cdot \nabla \varphi_{n}\right| & \leq C \iint_{Q_{1}(0,0)}|v| \cdot|\pi|+C \sum_{k=3}^{n-1} \frac{1}{r_{k}^{4}} \iint_{Q_{r_{k}}}|v| \cdot\left|\pi-\bar{\pi}_{k}\right| \\
& \leq C \iint_{Q_{1}(0,0)}|v| \cdot|\pi|+C \sum_{k=3}^{n-1} r_{k} l\left(r_{k}\right),
\end{aligned}
$$

where $\bar{\pi}_{k}$ is the average of $\pi$ on $B_{r_{k}}$.

In conclusion,

$$
\begin{aligned}
a\left(r_{n}\right)+2 d\left(r_{n}\right) \leq & C \iint_{Q_{1}(0,0)}\left(|v|^{2}+|v| \cdot|\pi|\right) \\
& +C \sum_{k=1}^{n} r_{k}\left(g\left(r_{k}\right)+g\left(r_{k}\right)^{\frac{2}{3}} g\left(z, r_{k}\right)^{\frac{1}{3}}+g\left(r_{k}\right)^{\frac{1}{3}} g\left(z, r_{k}\right)^{\frac{2}{3}}\right) \\
& +C \sum_{k=1}^{n} r_{k} d\left(r_{k}\right)^{\frac{1}{2}}\left(g\left(r_{k}\right)^{\frac{1}{3}} s\left(r_{k}\right)^{\frac{1}{6}}+z\left(r_{k}\right)^{\frac{1}{2}}\right) \\
& +C \sum_{k=3}^{n-1} r_{k} l\left(r_{k}\right) .
\end{aligned}
$$


The proof proceeds by induction to show that

$$
a\left(r_{n}\right)+d\left(r_{n}\right) \leq C_{0} \varepsilon_{1}^{2 / 3}, \quad n \geq 2 .
$$

If $n=2$, then (by the local energy inequality with a smooth function equal to 1 in $Q_{r_{2}}$ and with compact support in $Q_{r_{1}}$ )

$$
\begin{aligned}
a\left(r_{2}\right)+2 d\left(r_{2}\right) \leq & C \iint_{Q_{1}(0,0)}|v|^{2}+C \iint_{Q_{1}(0,0)}\left(|v|^{3}+|v|^{2}|z|+|z|^{2}|v|\right) \\
& +C \iint_{Q_{1}(0,0)}\left(|v| \cdot|z| \cdot|\nabla v|+|z|^{2}|\nabla v|\right)+C \iint_{Q_{1}(0,0)}|v| \cdot|\pi| \\
\leq & C\left(\varepsilon_{1}^{\frac{2}{3}}+\varepsilon_{1}+\varepsilon_{1}^{\frac{2}{3}} \varepsilon_{3}^{\frac{1}{q}}+\varepsilon_{1}^{\frac{1}{3}} \varepsilon_{3}^{\frac{1}{q}}+\varepsilon_{1}^{\frac{1}{3}} \varepsilon_{3}^{\frac{1}{q}} d\left(r_{1}\right)^{\frac{1}{2}}+\varepsilon_{3}^{\frac{1}{q}} d\left(r_{1}\right)^{\frac{1}{2}}\right) \\
\leq & C_{0} \varepsilon_{1}^{2 / 3}
\end{aligned}
$$

if $\varepsilon_{1}, \varepsilon_{2}$ and $\varepsilon_{3}$ are small enough.

Now, suppose the property holds true for $k=2, \ldots, n-1$. First,

$$
g\left(r_{k}\right) \leq C\left(a\left(r_{k}\right)+d\left(r_{k}\right)\right)^{3 / 2} \leq C C_{0}^{3 / 2} \varepsilon_{1} .
$$

The four other terms are estimated. The first

$$
C \iint_{Q_{1}(0,0)}\left(|v|^{2}+|v| \cdot|\pi|\right) \leq C\left(\varepsilon_{1}^{2 / 3}+\varepsilon_{1}\right) \leq \frac{1}{4} C_{0} \varepsilon_{1}^{2 / 3},
$$

then the second

$$
\begin{aligned}
& C \sum_{k=1}^{n} r_{k}\left(g\left(r_{k}\right)+g\left(r_{k}\right)^{\frac{2}{3}} g\left(z, r_{k}\right)^{\frac{1}{3}}+g\left(r_{k}\right)^{\frac{1}{3}} g\left(z, r_{k}\right)^{\frac{2}{3}}\right) \\
& \leq C \sum_{k=1}^{n} r_{k}\left(C_{0}^{\frac{3}{2}} \varepsilon_{1}+C_{0} r_{k}^{-\frac{3}{q}} \varepsilon_{1}^{\frac{2}{3}} \varepsilon_{3}^{\frac{1}{q}}+C_{0}^{\frac{1}{2}} r_{k}^{-\frac{6}{q}} \varepsilon_{1}^{\frac{1}{3}} \varepsilon_{3}^{\frac{2}{q}}\right) \\
& \quad \leq C\left(C_{0}^{\frac{3}{2}} \varepsilon_{1}+C_{0} \varepsilon_{1}^{\frac{2}{3}} \varepsilon_{3}^{\frac{1}{q}}+C_{0}^{\frac{1}{2}} \varepsilon_{1}^{\frac{1}{3}} \varepsilon_{3}^{\frac{2}{q}}\right) \\
& \quad \leq \frac{1}{4} C_{0} \varepsilon_{1}^{2 / 3} .
\end{aligned}
$$

Since

$$
g\left(r_{k}\right)^{\frac{1}{3}} s\left(r_{k}\right)^{\frac{1}{6}}+z\left(r_{k}\right)^{\frac{1}{2}} \leq C\left(C_{0}^{\frac{1}{2}} r_{k}^{-\frac{3}{q}} \varepsilon_{1}^{\frac{1}{3}} \varepsilon_{3}^{\frac{1}{q}}+r_{k}^{-\frac{6}{q}} \varepsilon_{3}^{\frac{2}{q}}\right),
$$

the third term is estimated using Young's inequality,

$$
\begin{aligned}
C \sum_{k=1}^{n} & r_{k} d\left(r_{k}\right)^{\frac{1}{2}}\left(g\left(r_{k}\right)^{\frac{1}{3}} s\left(r_{k}\right)^{\frac{1}{6}}+z\left(r_{k}\right)^{\frac{1}{2}}\right) \\
& \leq C C_{0}^{\frac{1}{2}} \varepsilon_{1}^{\frac{1}{3}}\left(C_{0}^{\frac{1}{2}} \varepsilon_{1}^{\frac{1}{3}} \varepsilon_{3}^{\frac{1}{q}}+\varepsilon_{3}^{\frac{2}{q}}\right)+C\left(C_{0}^{\frac{1}{2}} \varepsilon_{1}^{\frac{1}{3}} \varepsilon_{3}^{\frac{1}{q}}+\varepsilon_{3}^{\frac{2}{q}}\right) d\left(r_{n}\right)^{\frac{1}{2}} \\
& \leq C C_{0} \varepsilon_{1}^{\frac{2}{3}} \varepsilon_{3}^{\frac{1}{q}}+C C_{0}^{\frac{1}{2}} \varepsilon_{1}^{\frac{1}{3}} \varepsilon_{3}^{\frac{2}{q}}+C C_{0} \varepsilon_{1}^{\frac{2}{3}} \varepsilon_{3}^{\frac{2}{q}}+C \varepsilon_{3}^{\frac{4}{q}}+d\left(r_{n}\right) \\
& \leq \frac{1}{4} C_{0} \varepsilon_{1}^{2 / 3}+d\left(r_{n}\right) .
\end{aligned}
$$


Finally, the previous lemma is used to estimate the fourth term. In fact,

$$
\begin{aligned}
k\left(\frac{1}{4}\right) & \leq C \varepsilon_{1}, \\
g\left(v+z, \frac{1}{4}\right) & \leq C\left(g\left(\frac{1}{4}\right)+g\left(z, \frac{1}{4}\right)\right) \leq C\left(\varepsilon_{1}+\varepsilon_{3}^{3 / q}\right), \\
g\left(v+z, r_{k}\right) & \leq C\left(g\left(r_{k}\right)+g\left(z, r_{k}\right)\right) \leq C\left(C_{0}^{3 / 2} \varepsilon_{1}+r_{k}^{-3 / q} \varepsilon_{3}^{3 / q}\right),
\end{aligned}
$$

and

$$
\begin{aligned}
\int_{r_{k-1}<|y|<1 / 4} \frac{|v+z|^{2}}{|y|^{4}} & =\sum_{i=2}^{k-2} \int_{B_{r_{i}} \backslash B_{r_{i+1}}} \frac{|v+z|^{2}}{|y|^{4}} \\
& \leq C \sum_{i=2}^{k-2} \frac{1}{r_{i}^{4}} \int_{B_{r_{i}}}\left(|v|^{2}+|z|^{2}\right) \\
& \leq C \sum_{i=2}^{k-2}\left(\frac{1}{r_{i}} a\left(r_{i}\right)+\frac{1}{r_{i}^{1+\frac{6}{q}}} \varepsilon_{3}^{\frac{2}{q}}\right) \\
& \leq \frac{C}{r_{k-1}^{1+\frac{6}{q}}}\left(C_{0} \varepsilon_{1}^{2 / 3}+\varepsilon_{3}^{2 / q}\right) .
\end{aligned}
$$

By the previous lemma with $\rho=\frac{1}{4}$ and $r=r_{k}$, it follows that

$$
\begin{aligned}
l\left(r_{k}\right) \leq & C r_{k}^{\frac{5}{3}} g\left(r_{k}\right)^{\frac{1}{3}} g\left(v+z, \frac{1}{4}\right)^{\frac{2}{3}}+C g\left(r_{k}\right)^{\frac{1}{3}} g\left(v+z, r_{k-1}\right)^{\frac{2}{3}} \\
& +C r_{k} g\left(r_{k}\right)^{\frac{1}{3}} \sup _{-r_{k}^{2}<t<0} \int_{r_{k-1}<y<\frac{1}{4}} \frac{|v+z|^{2}}{|y|^{4}} d y+\frac{C}{r_{k}^{2}} r_{k}^{\frac{7}{5}} a\left(r_{k}\right)^{\frac{1}{5}} g\left(r_{k}\right)^{\frac{1}{5}} k\left(\frac{1}{4}\right)^{\frac{4}{5}} \\
\leq & C C_{0}^{\frac{1}{2}} \varepsilon_{1}^{\frac{1}{3}}\left(r_{k}^{\frac{5}{3}}\left(\varepsilon_{1}^{\frac{2}{3}}+\varepsilon_{3}^{\frac{2}{q}}\right)+C_{0} \varepsilon_{1}^{\frac{2}{3}}+r_{k}^{-\frac{2}{q}} \varepsilon_{3}^{\frac{2}{q}}+r_{k}^{-\frac{6}{q}}\left(C_{0} \varepsilon_{1}^{\frac{2}{3}}+\varepsilon_{3}^{\frac{2}{q}}\right)\right) \\
& +C C_{0}^{\frac{1}{2}} r_{k}^{-\frac{3}{5}} \varepsilon_{1} \\
\leq & C\left(C_{0} \varepsilon_{1}+C_{0} \varepsilon_{1}^{\frac{1}{3}} \varepsilon_{3}^{\frac{2}{3}}+r_{k}^{-\frac{6}{q}} \varepsilon_{1}^{\frac{1}{3}} \varepsilon_{3}^{\frac{2}{q}}+C_{0}^{\frac{1}{2}} r_{k}^{-\frac{3}{5}} \varepsilon_{1}\right)
\end{aligned}
$$

and so

$$
C \sum_{k=3}^{n-1} r_{k} l\left(r_{k}\right) \leq C\left(C_{0}^{3 / 2} \varepsilon_{1}+C_{0} \varepsilon_{1}^{1 / 3} \varepsilon_{3}^{2 / q}\right) \leq \frac{1}{4} C_{0} \varepsilon_{1}^{2 / 3} .
$$

All the previous inequalities worked since the various constants were chosen in the following way:

$$
C_{0} \geq 8 C, \quad 2 C_{0}^{3 / 2} \varepsilon_{1}^{1 / 3} \leq 1, \quad \varepsilon_{3}^{1 / q} \leq \varepsilon_{1}, \quad \varepsilon_{2} \leq 1 .
$$

In conclusion,

$$
a\left(r_{n}\right)+2 d\left(r_{n}\right) \leq C_{0} \varepsilon_{1}^{2 / 3}+d\left(r_{n}\right)
$$

and so

$$
a\left(r_{n}\right)+d\left(r_{n}\right) \leq C_{0} \varepsilon_{1}^{2 / 3} .
$$


The main result on the local regularity is now deduced by the results of this section and the results of the previous section. In addition, an argument of scaling invariance is used to complete the proof.

Theorem 5.3. Assume (4.1) and let $(u, P)$ be a suitable weak solution. Let $(z, Q)$ be the solution of the Stokes system, and let $(v, \pi)$ be obtained as in (2.2). Then there exists $\varepsilon>0$ (depending on $z$ ) such that the inequality

$$
\limsup _{r \rightarrow 0} \frac{1}{r} \iint_{Q_{r}(0,0)}|\nabla v|^{2} \leq \varepsilon
$$

implies that $(0,0)$ is a regular point for $v$.

Proof. Let $\varepsilon_{1}, \varepsilon_{2}$ and $\varepsilon_{3}$ be the three constants given by the previous proposition. According to Theorem 4.2 corresponding to $\varepsilon_{1}$ there exists $\varepsilon \leq \varepsilon_{2}$ such that $\lim \sup _{r \rightarrow 0} \delta_{*}(r) \leq \varepsilon$ implies

$$
\limsup _{r \rightarrow 0}\left\{G_{*}(r)+J_{*}(r)+K_{*}(r)\right\} \leq \frac{1}{2} \varepsilon_{1}
$$

and in addition from (5.1) it follows that

$$
\limsup _{r \rightarrow 0} r^{q-3} \sup _{-r^{2}<t<0} \int_{B_{r}}|z|^{q} d x=0 .
$$

Rescaling $v$ and $z$ (they are still suitable weak solutions ...), we get

$$
\begin{aligned}
\iint_{Q_{1}}\left(\left|v_{\lambda}\right|^{3}+\left|v_{\lambda}\right| \cdot\left|\pi_{\lambda}\right|\right) d x d t+\int_{-1}^{0}\left(\int_{B_{1}}\left|\pi_{\lambda}\right| d x\right)^{5 / 4} d t & \leq \varepsilon_{1}, \\
\iint_{Q_{1}}\left|\nabla v_{\lambda}\right|^{2} d x d t & \leq \varepsilon_{2}, \\
\sup _{-1<t<0} \int_{B_{1}}\left|z_{\lambda}\right|^{q} d x & \leq \varepsilon_{3}
\end{aligned}
$$

for some $\lambda>0$. By the previous proposition, it follows that $(0,0)$ is a regular point for the rescaled solution, hence for the solution itself. This proves the claim.

The previous theorem asserts the regularity of the point $(0,0)$ for the function $v=u-z$, and not for the function $u$. The regularity for $u$ follows from the regularity of $z$, which is not included in the previous assumption. We can then deduce Proposition 3.1 ,

Corollary 5.4. Assume $g \equiv 0$ and

$$
f \in L^{p}((0, T) \times D) \cap L^{2}(0, T ; H) .
$$

Let $(u, P)$ be a suitable weak solution and let $S$ be the set of singular points of $u$.

If $p>\frac{5}{2}$, then $\mathcal{H}^{1}(S)=0$.

If $p \in\left(2, \frac{5}{2}\right]$, then for a.e. $t \in[0, T]$ we have $u(t) \in L_{l o c}^{\infty}(D)$, and moreover there exists $D_{0} \subset D$ such that $\mathcal{H}^{1}\left(D_{0}\right)=0$ and for each $x \in D \backslash D_{0}$

$$
\sup _{t \in(0, T)}|u(t, x)|<\infty .
$$

Proof. This corollary is an easy consequence of the previous theorem, a standard covering argument and results on the regularity of $z$ proved in the appendix. 


\section{REGULARITY OF STATIONARY SOLUTIONS}

Suppose we have a stationary measure $\mu$ with finite dissipation rate, that is,

$$
\int_{\mathcal{S}}\left[\int_{0}^{T} \int_{D}|\nabla u|^{2} d x d t\right] \mu(d(u, B))<\infty
$$

and denote by $\left(u_{s t}, B\right)$ the canonical process having $\mu$ as its law. Then all the trajectories of $u_{s t}$ are suitable weak solutions in the sense of Definition 2.5. We will assume throughout this section that

$$
\begin{aligned}
& \text { the marginal of } \mu \text { in the second component } \\
& \text { is concentrated on } C^{\frac{1}{2}-\varepsilon}\left([0, \infty) ; D\left(A^{\frac{1}{4}+\beta}\right)\right)
\end{aligned}
$$

for some $\beta>\varepsilon>0$.

The aim of this section is to study the regularity of the trajectories of this process. Denote by $S_{t}(u)$ the set of singular points of the function $u$ at time $t$, that is, the singular points of the form $(t, x)$.

Theorem 6.1. Assume 6.1). For any $t_{0}$,

$$
S_{t_{0}}\left(u_{s t}\right)=\emptyset
$$

with probability one.

Proof. If

$$
\lim _{r \rightarrow 0} \frac{1}{r} \int_{t_{0}-r^{2}}^{t_{0}+r^{2}} \int_{D}\left|\nabla u_{s t}\right|^{2} d x d t=0
$$

with probability one, then by virtue of Theorem 5.3, each point $\left(t_{0}, x\right)$ is regular. Let $r_{n}=2^{-n}$; then

$$
\frac{1}{r} \int_{t_{0}-r^{2}}^{t_{0}+r^{2}} \int_{D}\left|\nabla u_{s t}\right|^{2} d x d t \leq 2 \frac{1}{r_{n}} \int_{t_{0}-r_{n}^{2}}^{t_{0}+r_{n}^{2}} \int_{D}\left|\nabla u_{s t}\right|^{2} d x d t
$$

if $r \in\left(r_{n+1}, r_{n}\right)$, and so it is sufficient to show that the sequence of random variables

$$
X_{n}=\frac{1}{r_{n}} \int_{t_{0}-r_{n}^{2}}^{t_{0}+r_{n}^{2}} \int_{D}\left|\nabla u_{s t}\right|^{2} d x d t
$$

goes to zero with probability one. Since $u_{s t}$ has finite dissipation rate, it follows that

$$
\mathbf{E} X_{n}=C_{\mu} r_{n}
$$

for any fixed $\delta>0$,

$$
\mathbf{P}\left(X_{n}>\delta\right) \leq \frac{C_{\mu}}{\delta} r_{n}
$$

and so

$$
\sum \mathbf{P}\left(X_{n}>\delta\right)<\infty
$$

By the Borel-Cantelli lemma there exists a set $\Omega_{\delta}$ of full probability such that for each $\omega \in \Omega_{\delta}$ there exists $n(\omega)$ such that $X_{n}(\omega)<\delta$ for all $n>n(\omega)$. The conclusion follows by taking a sequence $\delta_{k} \rightarrow 0$. 
Remark 6.2. It seems that these techniques do not allow us to prove a stronger result, namely

$$
S\left(u_{\mathrm{st}}\right)=\emptyset
$$

with probability one. In [9] a counterexample is presented to show that the ingredients we used in the previous proof are not sufficient to obtain the best result.

Remark 6.3. Other invariances can be treated with the same techniques. For example, if we are on the full space or on a torus, it would be interesting to study homogeneous (that is, space-invariant) solutions. Then we could have

$$
\mathbf{E} \int_{0}^{T} \int_{B_{r}(x)}|\nabla u|^{2} d x d t=C r^{3}
$$

and consequently a result similar to Theorem 6.1 for a given $x_{0} \in \mathbf{R}^{3}$ instead of a given $t_{0} \geq 0$.

The end of this section will be devoted to the proof of Corollary 3.6 Prior to this, we need to show that a stationary solution can be decomposed conditional to the initial datum.

The following lemma will let us consider a stationary solution as a measure on the space $L_{\text {loc }}^{2}(0, \infty ; H) \times C_{0}([0, \infty) ; H)$.

Lemma 6.4. The set $\mathcal{S}$ is a Borel subset of $L_{\mathrm{loc}}^{2}(0, \infty ; H) \times C_{0}([0, \infty) ; H)$.

Proof. Let

$$
\mathcal{R}=L_{\text {loc }}^{2}([0, \infty) ; H) \times C_{0}([0, \infty) ; H)
$$

and fix $0<\varepsilon<\delta, T>0$ and $M>0$. Consider the subset $\mathcal{S}_{\delta, \varepsilon, T, M}$ of $\mathcal{R}$ of functions

$$
(u, B) \in\left[L_{\mathrm{loc}}^{\infty}((0, T] ; H) \cap L_{\mathrm{loc}}^{2}((0, T] ; V)\right] \times C^{\frac{1}{2}-\varepsilon}\left([0, \infty) ; D\left(A^{\delta}\right)\right)
$$

such that

$$
\|B\|_{C^{\frac{1}{2}-\varepsilon}\left([0, T] ; D\left(A^{\delta}\right)\right)} \leq M
$$

where $A$ is the operator defined in Section 1.1 If $u=v+z$ and $P, \pi$ and $Q$ are the related pressure fields, then $(z, Q)$ solve the Stokes equations and $(v, \pi)$ solve the modified Navier-Stokes equations and fulfill the local energy inequality and the classical energy inequality.

The lemma is proved if each set $\mathcal{S}_{\delta, \varepsilon, T, M}$ is closed in $\mathcal{R}$, since it is easy to see that $\mathcal{S}$ is equal to

$$
\left[\bigcap_{T>0} \bigcup_{M>0} \bigcup_{0<\varepsilon<\delta} \mathcal{S}_{\delta, \varepsilon, T, M}\right] \cap\left[\left(C\left([0, \infty) ; H_{w}\right) \cap L_{\mathrm{loc}}^{2}([0, \infty) ; V)\right) \times C_{0}([0, \infty) ; H)\right]
$$

and, if $T_{n} \uparrow \infty, M_{n} \uparrow \infty, \varepsilon_{n} \downarrow 0$ and $\delta_{n} \downarrow 0$, the conclusion is that $\mathcal{S}$ is a Borel subset of $\mathcal{R}$.

The sets $\mathcal{S}_{\delta, \varepsilon, T, M}$ are closed. In fact, let $\left(u_{N}, B_{N}\right)$ in $\mathcal{S}_{\delta, \varepsilon, T, M}$ be convergent in $\mathcal{R}$ to some $(u, B)$. Write $u_{N}=v_{N}+z_{N}$; by the bound on $B_{N}$ it follows that $B \in C^{\frac{1}{2}-\varepsilon}\left([0, T] ; D\left(A^{\delta}\right)\right)$ and that $z_{N}$ converge to the solution $z$ of Stokes equations with forcing term the time derivative of $B$. So to prove that $(u, B) \in \mathcal{S}_{\delta, \varepsilon, T, M}$, it is sufficient to show that $v_{N}$ are bounded uniformly in $N$ in

$$
L^{\infty}(a, T ; H) \cap L^{2}(a, T ; V)
$$


for each $a>0$. Fix $a>0$; then by the classical energy inequality, using the Sobolev inequality and the Young inequality,

$$
\begin{aligned}
\left|v_{N}(t)\right|^{2}+2 \int_{s}^{t}\left|\nabla v_{N}\right|^{2} \leq & \left|v_{N}(s)\right|^{2}+2 \int_{s}^{t} z_{N} \cdot\left(\left(v_{N}+z_{N}\right) \cdot \nabla\right) v_{N} \\
\leq & \left|v_{N}(s)\right|^{2}+\int_{s}^{t}\left|\nabla v_{N}\right|^{2}+C \int_{s}^{t}\left\|z_{N}\right\|_{L^{4}(D)}^{8} \\
& +C \int_{s}^{t}\left|v_{N}\right|^{2}\left\|z_{N}\right\|_{L^{4}}^{8} .
\end{aligned}
$$

Since $\left\|z_{N}\right\|_{L^{4}(D)}$ and $\left\|v_{N}\right\|_{L^{2}\left(D_{T}\right)}$ are uniformly bounded in $N$, if the previous inequality is integrated in $s \in(0, t)$ and the fact that $t \geq a$ is used, then

$$
\begin{aligned}
a\left|v_{N}(t)\right|^{2}+a \int_{a}^{t}\left|\nabla v_{N}\right|^{2} & \leq t\left|v_{N}(t)\right|^{2}+\int_{0}^{t} \int_{s}^{t}\left|\nabla v_{N}\right|^{2} \\
& \leq \int_{0}^{t}\left|v_{N}\right|^{2}+C_{M} t+C_{M} \int_{0}^{t} \int_{s}^{t}\left|v_{N}\right|^{2} \\
& \leq C(M, T),
\end{aligned}
$$

and the claim follows.

Let $\nu$ be the marginal measure of the stationary solution $\mu$ in the $u$-variable and let $\nu_{t}$ be the image measure in $H$ of $\nu$ through the projection at time $t$. Notice that $\nu_{t}=\mu_{0}$ for all $t$. Then

$$
\mu(\cdot)=\int_{H} \mu\left(\cdot \mid u(0)=u_{0}\right) \nu_{0}\left(d u_{0}\right)
$$

so that

$$
\nu_{t}(\cdot)=\int_{H}\left[\nu\left(\cdot \mid u(0)=u_{0}\right)\right]_{t} \nu_{0}\left(d u_{0}\right)
$$

where $\mu\left(\cdot \mid u(0)=u_{0}\right)$ are, for a.e. $u_{0} \in H$, probability measures on $L_{\text {loc }}^{2}(0, \infty ; H)$ obtained by conditioning $\nu$ to the event $u(0)=u_{0}$ and $\left[\nu\left(\cdot \mid u(0)=u_{0}\right)\right]_{t}$ are the image measures at time $t$ of the conditional measures obtained above. Now we can deduce the proof of Corollary 3.6 .

Proof. Fix $t \in(0, \infty)$. By the previous theorem, the set $\mathcal{R}_{t}$ of trajectories which have no singular points at time $t$ has $\mu$-measure one. Let $u_{0} \in H$ and let $\left(u\left(\cdot, u_{0}\right), B\right)$ be the canonical process having $\mu\left(\cdot \mid u(0)=u_{0}\right)$ as its law. The process $u\left(\cdot, u_{0}\right)$ is a martingale suitable weak solution. Moreover, since by (6.2)

$$
\int_{H} \mu\left(\mathcal{R}_{t} \mid u(0)=u_{0}\right) \mu_{0}\left(d u_{0}\right)=\mu\left(\mathcal{R}_{t}\right)=1,
$$

it follows that

$$
\mu\left(\mathcal{R}_{t} \mid u(0)=u_{0}\right)=1 \quad \mu_{0} \text {-a.s. }
$$

In other words, $u\left(\cdot, u_{0}\right) \in \mathcal{R}_{t}$ a.s. for $\mu_{0}$-almost all $u_{0} \in H$. 
6.1. Effects of the noise on stationary solutions. The stirring effect of a sufficiently rich noise forces stationary solutions to visit all the space at any time. The consequence, stated in Theorem [3.7] is that the support of the measure $\mu_{0}$ on the initial data is the whole space $H$. Therefore the set of initial conditions $u_{0}$ with the regularity properties stated in Corollary 3.6 is quite rich. This claim can be seen using the result of irreducibility for the equations (2.1), proved by Flandoli in [8], under suitable assumptions on the noise. The theorem stated there applies to solutions of Navier-Stokes equations fulfilling pathwise the classical energy inequality

$$
|v(t)|^{2}+2 \int_{0}^{t}\|v(s)\|^{2} d s \leq\left|u_{0}\right|^{2}+2 \int_{0}^{t}\langle B(u(s), v(s)), u(s)\rangle d s+\int_{0}^{t}\langle f, v(s)\rangle d s,
$$

where, as usual, $v=u-z$. The paths of a martingale suitable weak solution, and hence of a stationary solution, fulfill the classical energy inequality (see [19]).

The law at time $t$ of a martingale solution starting in $u_{0}$ will be denoted by $p\left(t, u_{0}, \cdot\right)$. For the definition of the space $V$, see Section 1.1

Definition 6.5. The process $u$ is $(V-H)$-irreducible if for each $u_{0} \in V, t \in(0, T]$, $x \in H$ and $\varepsilon>0$

$$
p\left(t, u_{0}, B_{\varepsilon}^{H}\left(x_{0}\right)\right)>0,
$$

where $B_{\varepsilon}^{H}\left(x_{0}\right)$ is the ball of radius $\varepsilon$ and centre $x_{0}$ in $H$.

The condition $u_{0} \in V$ is not restrictive, since stationary solutions have finite dissipation rate; hence

$$
\mathbf{P}\left[u_{\mathrm{st}}(t) \in V\right]=1
$$

for every $t \geq 0$.

A sufficient condition that ensures the irreducibility is that the law of $B$ is full:

$$
\mathbf{P}\left[\|B-w\|_{W^{s, p}(0, T ; H)}<\varepsilon\right]>0
$$

for each $\varepsilon>0$ and $w \in W^{s, p}(0, T ; H)$, for some $s \in\left(0, \frac{1}{2}\right)$ and $p>1$ such that $s-\frac{1}{p}>\frac{3}{8}$. Moreover, the above property is assured, in the framework of Remark 2.4, if the covariance $\mathcal{O}$ in $H$ of $B$ is injective.

The main theorem of [8] is that, under the above assumption on the noise, every solution starting at $u_{0} \in V$ is $(V-H)$-irreducible. This result is now used to show a similar result for stationary solutions. More precisely, we want to show that for each $t \geq 0, \varepsilon>0$ and $x_{0} \in H$

$$
\mathbf{P}\left[u_{\mathrm{st}}(t) \in B_{\varepsilon}^{H}\left(x_{0}\right)\right]>0 .
$$

We use some of the notation of the previous section: the measure $\nu$ is the marginal of the stationary solution $\mu$ in the second component, while $\left[\nu\left(\cdot \mid u(0)=u_{0}\right)\right]_{t}$ is the measure $\nu$ conditional to the initial datum at time $t$.

Let $\left(u\left(\cdot, u_{0}\right), B\right)$ be the canonical random variable having $\mu\left(\cdot \mid u(0)=u_{0}\right)$ as its law. It is easy to see that $B$ is a Brownian motion which is full in the space in the sense of (6.4). Moreover, since

$$
\mu\left(\mathcal{S} \mid u(0)=u_{0}\right)=1,
$$

it follows easily that $u\left(\cdot, u_{0}\right)$ is a $(V-H)$-irreducible solution for all $T>0$. Notice that $\nu_{0}(V)=1$, and so, for all $\varepsilon>0, x_{0} \in H$ and $\nu_{0}$-a.a. $u_{0}$,

$$
\left[\nu\left(\cdot \mid u(0)=u_{0}\right)\right]_{t}\left(B_{\varepsilon}^{H}\left(x_{0}\right)\right)>0,
$$


since $\nu\left(\cdot \mid u(0)=u_{0}\right)$ is the law of $u\left(\cdot, u_{0}\right)$. Then by (6.3)

$$
\nu_{t}\left(B_{\varepsilon}^{H}\left(x_{0}\right)\right)=\int_{H}\left[\nu\left(\cdot \mid u(0)=u_{0}\right)\right]_{t}\left(B_{\varepsilon}^{H}\left(x_{0}\right)\right) \nu_{0}\left(d u_{0}\right)>0 .
$$

In conclusion, Theorem 3.7 holds true.

\section{Appendix A. Regularity for the Stokes system}

We prove the results of Section 2.2. In the first part we show that the assumptions on the mean force $f$ give the conclusions of Lemma 2.1 and 2.2. In the second part the proof of the two lemmas is completed. Finally, in the third part, we consider the stochastic Stokes system and we show that the conclusions of Lemma 2.1] and Lemma 2.2 hold almost surely for the process solution of the stochastic equation (see Remarks 2.3 and 2.4).

A.1. Regularity under a deterministic forcing term. In this section we will show the part of Lemmas 2.1 and 2.2 concerning the deterministic term $f$. Fix $T>0$ and consider the Stokes equation

$$
\frac{\partial z}{\partial t}+\nabla Q-\triangle z=f
$$

Assume first that $f \in L^{p}((0, T) \times D)$ for some $p>2$. Then there exists a solution $z$ such that

$$
z \in C\left([0, T] ; H^{1}(D)\right) \cap L^{p}\left(0, T ; W^{2, p}(D)\right), \quad \frac{\partial z}{\partial t} \in L^{p}((0, T) \times D) .
$$

From this it follows that $z \in L^{\infty}\left(0, T ; W^{1, p}(D)\right)$, so that

$$
z \in L^{\infty}\left(0, T ; L^{q}(D)\right) \quad \text { for some } q>6 \text {. }
$$

Moreover, clearly $z \in L^{\infty}\left(0, T ; L^{4}(D)\right) \cap L^{2}\left(0, T ; H_{0}^{1}(D)\right)$ and

$$
\frac{1}{r} \int_{t-r^{2}}^{t+r^{2}} \int_{B_{r}(x)}|\nabla z|^{2} \leq r \sup _{\left(t-r^{2}, t+r^{2}\right)} \int_{B_{r}(x)}|\nabla z|^{2} \leq C r \rightarrow 0 .
$$

Also, the regularity for the pressure is classical (see for example Solonnikov [31]). In conclusion, Lemma 2.1 is true.

Finally, Lemma 2.2 is shown. Moreover. in view of Proposition 3.1, the case $p \leq \frac{5}{2}$ is considered too.

Proposition A.1. If $p>\frac{5}{2}$, then $z$ is essentially bounded in all $D \times[0, T]$.

If $p \in\left(2, \frac{5}{2}\right]$, then $z(t) \in C(D)$ for a.e. $t \in[0, T]$, and there exists a subset $D_{0} \subset D$ with one-dimensional Hausdorff measure equal to zero such that for each $x \in D \backslash D_{0}$

$$
\sup _{0<t<T}|z(t, x)|<\infty .
$$

Proof. The first part of the proposition is a classical result.

From the regularity theory of the heat equation it is known that

$$
z \in L^{p}\left(0, T ; W^{2, p}(D)\right),
$$

and then, by the Sobolev inequalities, $z(t) \in C(D)$ for a.e. $t \in[0, T]$. The second part of the proposition now follows from Theorem 5.3 of Temam [34]. 
Remark A.2. It is possible to ask (and it has been done when stationary solutions have been studied) about different summability conditions on $f$ to obtain a bounded $z$. In fact it can be shown that if

$$
f \in L^{p_{1}}\left(0, T ; L^{p_{2}}(D)\right), \quad \text { with } p_{1}, p_{2}>\frac{3}{2}, \quad \frac{2}{p_{1}}+\frac{3}{p_{2}}<2,
$$

then $z$ is essentially bounded.

Define the maximal function of $f$,

$$
f^{*}(t, x)=\sup _{r} \frac{1}{r^{5}} \iint_{Q_{r}(t, x)}|f(s, y)| d s d y
$$

(see Stein [32] for more details).

Lemma A.3. If $f \in L^{p_{1}}\left(0, T ; L^{p_{2}}\left(\boldsymbol{R}^{3}\right)\right)$, then $f^{*} \in L^{p_{1}}\left(0, T ; L^{p_{2}}\left(\boldsymbol{R}^{3}\right)\right)$ and

$$
\left\|f^{*}\right\|_{L^{p_{1}}\left(0, T ; L^{p_{2}}\left(\boldsymbol{R}^{3}\right)\right)} \leq C_{p_{1}, p_{2}}\left\|f^{*}\right\|_{L^{p_{1}}\left(0, T ; L^{p_{2}}\left(\boldsymbol{R}^{3}\right)\right)} .
$$

Proof. The lemma is an easy consequence of the maximal theorem. Fix $t \in[0, T]$ and set

$$
f^{\#}(t, x)=\sup _{b>0} \frac{1}{b^{3}} \int_{B_{b}(x)}|f(t, y)| d y, \quad x \in \mathbf{R}^{3} .
$$

Since $f(t, \cdot) \in L^{p_{2}}\left(\mathbf{R}^{3}\right)$ for a.e. t, then $f^{\#}(t, \cdot) \in L^{p_{2}}\left(\mathbf{R}^{3}\right)$ by the maximal theorem, and

$$
\left\|f^{\#}(t)\right\|_{L^{p_{2}}\left(\mathbf{R}^{3}\right)} \leq C_{p_{2}}\|f(t)\|_{L^{p_{2}}\left(\mathbf{R}^{3}\right)},
$$

and so $f^{\#} \in L^{p_{1}}\left(0, T ; L^{p_{2}}\left(\mathbf{R}^{3}\right)\right)$. Now the same is done with a fixed $x \in \mathbf{R}^{3}$ to $f^{\#}$; hence the function

$$
f^{\# \#}(t, x)=\sup _{a>0} \frac{1}{a} \int_{t-a}^{t+a}\left|f^{\#}(s, x)\right| d s
$$

is in $L^{p_{1}}\left(0, T ; L^{p_{2}}\left(\mathbf{R}^{3}\right)\right)$. Finally, the conclusion follows since $f^{*} \leq f^{\# \#}$.

With the aid of this lemma the local boundedness of $z$ is proved, if $f$ satisfies locally the property (A.1) (the proof is similar to that of Theorem 5.3 in Temam [34]). Without loss of generality it is assumed that $D=\mathbf{R}^{3}$, and that $z$ and $f$ have compact support (otherwise a cut-off function is used).

Proposition A.4. Suppose that $f$ satisfies (A.1) in $(0, T) \times \boldsymbol{R}^{3}$. Then $z$ is locally bounded.

Proof. It is known that for a.e. $(t, x) \in \mathbf{R}^{3} \times(0, T)$

$$
z(t, x)=\int_{0}^{t} \int_{\mathbf{R}^{3}} E(s, y) f(t-s, x-y) d s d y
$$

where $E(s, y)$ is the heat kernel.

For fixed $\left(t_{0}, x_{0}\right) \in(0, T) \times \mathbf{R}^{3}$, an application of Fubini and the change of variables $\left(t^{\prime}, x^{\prime}\right)=(t-s, x-y)$ give

$$
\frac{1}{r^{5}} \iint_{Q_{r}\left(t_{0}, x_{0}\right)}|z(t, x)| d t d x \leq \int_{0}^{t_{0}} \int_{\mathbf{R}^{3}} E(s, y) f^{*}\left(t_{0}-s, x_{0}-y\right) d y d s .
$$


Now, for any $R>0$,

$$
\begin{aligned}
\int_{0}^{t_{0}} & \int_{\mathbf{R}^{3}} E(s, y) f^{*}\left(t_{0}-s, x_{0}-y\right) d y d s \\
& =\iint_{\left(0, t_{0}\right) \times \mathbf{R}^{3} \backslash Q_{R}\left(t_{0}, x_{0}\right)} E\left(t_{0}-s, x_{0}-y\right) f^{*}(s, y) d y, d s \\
& +\sum_{j=0}^{\infty} \iint_{Q_{R / 2} j\left(t_{0}, x_{0}\right) \backslash Q_{R / 2^{j+1}}\left(t_{0}, x_{0}\right)} E\left(t_{0}-s, x_{0}-y\right) f^{*}(s, y) d y d s \\
& =\mathrm{I}+\mathrm{II} .
\end{aligned}
$$

From estimates on the heat kernel, it follows that

$$
\mathrm{I} \leq C_{R, p_{1}, p_{2}, T}\left\|f^{*}\right\|_{L^{p_{1}}\left(0, T ; L^{p_{2}}\left(\mathbf{R}^{3}\right)\right)},
$$

while

$$
\mathrm{II} \leq \sum_{j=0}^{\infty} C\left(\frac{2^{j}}{R}\right)^{3-\frac{3}{p_{2}}-\frac{2}{p_{1}^{\prime}}}\left\|f^{*}\right\|_{L^{p_{1}}\left(0, T ; L^{p_{2}}\left(\mathbf{R}^{3}\right)\right.},
$$

since by the assumption (A.1) $3-\frac{3}{p_{2}^{\prime}}-\frac{2}{p_{1}^{\prime}}<0$, where $p_{1}^{\prime}, p_{2}^{\prime}$ are the Hölder conjugate exponents of $p_{1}, p_{2}$.

Finally, if $p_{1}=\infty$ the proof proceeds in a similar way.

A.2. Regularity under a non-regular deterministic forcing term. Consider the Stokes equation in a bounded domain $D$ :

$$
\frac{\partial z}{\partial t}-\triangle z+\nabla Q=\frac{\partial g}{\partial t}
$$

When $g$ is differentiable in a suitable sense, the solution is given by

$$
z(t)=\int_{0}^{t} e^{-(t-s) A} d g(s) .
$$

However, it will only be assumed that $g$ is Hölder continuous in time, so the previous integral has to be properly defined. This has been done in [7], and the following result has been proved.

Proposition A.5. If $g \in C^{\alpha}\left([0, T] ; D\left(A^{\beta}\right)\right)$, then there exists a unique solution of the Stokes system such that for all $\varepsilon>0$,

$$
z \in C\left([0, T] ; D\left(A^{\alpha+\beta-\varepsilon}\right)\right) .
$$

We explain now in which sense $Q$ is, together with $z$, a solution of (A.2). Let $J_{n}=n(n-A)^{-1}$ be the Yosida approximations, having the properties

$$
\begin{aligned}
J_{n}(H) & =D(A), \quad J_{n} A=A J_{n} \text { on } D(A), \\
J_{n} x & \rightarrow x \text { as } n \rightarrow \infty \text { for every } x \in H .
\end{aligned}
$$

From the integral equation for $z$, applying the operator $J_{n}$ to each term, it is not difficult to prove that

$$
J_{n} z(t)=\int_{0}^{t} A J_{n} z(s) d s+J_{n} g(t) .
$$


Moreover, by the classical Helmholtz decomposition in $L^{2}(D)$, for every $n \in \mathbf{N}$ and $t \geq 0$, there exists $Q_{n}(t) \in H^{1}(D)$, unique up to a constant, such that

$$
A J_{n} z(t)=\triangle J_{n} z(t)+\nabla Q_{n}(t) .
$$

One can also choose $Q_{n}(t)$ in such a way that $Q_{n} \in L^{\infty}\left(0, T ; H^{1}(D)\right)$ for all $T \geq 0$, since $z \in L^{\infty}(0, T ; H)$. Therefore

$$
J_{n} z(t)=\int_{0}^{t}\left(\triangle J_{n} z(s)+\nabla Q_{n}(s)\right) d s+J_{n} g(t) .
$$

Now, for every $\phi \in C_{0}^{\infty}([0, T] \times D)$, from equation (A.4) we have

$$
\left\langle\triangle J_{n} z(t), \mathcal{P} \phi(t)\right\rangle_{L^{2}(D)}=\left\langle\triangle J_{n} z(t), \phi(t)\right\rangle_{L^{2}(D)}+\left\langle\nabla Q_{n}(t), \phi(t)\right\rangle_{L^{2}(D)},
$$

and thus, first integrating by parts and then integrating in time, it follows that

$$
\left\langle J_{n} z, \triangle \mathcal{P} \phi\right\rangle_{L^{2}([0, T] \times D)}=\left\langle J_{n} z, \triangle \phi\right\rangle_{L^{2}([0, T] \times D)}+\left\langle Q_{n}, \operatorname{div} \phi\right\rangle_{L^{2}([0, T] \times D)} .
$$

It follows that $Q_{n}$ converges weak star in $L^{\infty}\left(0, T ; H^{-1}(D)\right)$ to some $Q$. So we pass to the limit in (A.5) and get

$$
\begin{aligned}
\langle z(t), \varphi\rangle_{L^{2}(D)}= & \int_{0}^{t}\left(\langle z(s), \triangle \varphi\rangle_{L^{2}(D)}+\langle Q(s), \operatorname{div} \varphi\rangle_{L^{2}(D)}\right) d s \\
& +\langle g(t), \varphi\rangle_{L^{2}(D)}
\end{aligned}
$$

for all $\phi \in C_{0}^{\infty}(D)$.

Now, with the help of these considerations, it is possible to complete the proof of Lemma 2.1 By Proposition A.5 and by the Sobolev embeddings (1.4), if $g \in$ $C^{\frac{1}{2}-\varepsilon}\left([0, T] ; D\left(A^{\beta}\right)\right)$, then $z \in C\left([0, T] ; D\left(A^{\frac{1}{2}+\beta-\varepsilon}\right)\right)$, and so, for a $q>6$,

$$
D\left(A^{\frac{1}{2}+\beta-\varepsilon}\right) \subset H^{1+2(\beta-\varepsilon)} \subset L^{q}(D) .
$$

The regularity of $Q$ follows from A.6 and from classical estimates (see for example Solonnikov [31).

Finally, also Lemma 2.2 follows, since if $g \in C^{\frac{1}{2}-\varepsilon}\left([0, T] ; D\left(A^{\frac{1}{4}+\beta}\right)\right)$ then we have $z \in C\left([0, T] ; D\left(A^{\frac{3}{4}+\beta-\varepsilon}\right)\right)$, and consequently,

$$
D\left(A^{\frac{3}{4}+\beta-\varepsilon}\right) \subset H^{\frac{3}{2}+2(\beta-\varepsilon)} \subset L^{\infty}(D) .
$$

A.3. Regularity under a stochastic forcing term. In this last section we consider the problem of the stochastic Stokes system in the bounded domain $D$ :

$$
d z-\triangle z d t+\nabla Q d t=\sqrt{\mathcal{O}} d W .
$$

Using the framework of Remark 2.4 we assume that $W_{t}$ is a cylindrical Wiener process in $H$, and $\mathcal{O}$ is a positive self-adjoint bounded operator in $H$, with suitable further regularity. The solution is given by the stochastic integral

$$
z(t)=\int_{0}^{t} e^{-(t-s) A} \sqrt{\mathcal{O}} d W_{s}
$$

defined in Da Prato and Zabczyk [5]. It gives a continuous process $z$ in $H$. A formal proof (which can be easily made rigorous), outlined in the sequel, shows that in the stochastic setting the conclusions of Lemmas 2.1 and 2.2 hold almost surely. We prove the claim of Lemma 2.1 under the assumption

$$
\operatorname{Tr}\left[A^{\varepsilon} \mathcal{O} A^{\varepsilon}\right]<\infty \quad \text { for some } \varepsilon>0
$$


(see Remarks 2.3 and 2.4). The argument can be easily adapted to show the claim of Lemma 2.2 under the assumption

$$
\operatorname{Tr}\left[A^{\frac{1}{4}+\varepsilon} \mathcal{O} A^{\frac{1}{4}+\varepsilon}\right]<\infty \quad \text { for some } \varepsilon>0 .
$$

The operator $A^{\frac{1}{2}+\frac{\varepsilon}{2}}$ is applied formally to get

$$
A^{\frac{1}{2}+\frac{\varepsilon}{2}} z(t)=\int_{0}^{t} A^{\frac{1}{2}-\frac{\varepsilon}{2}} e^{-(t-s) A} A^{\varepsilon} \sqrt{\mathcal{O}} d W_{s} .
$$

The criterion of Da Prato and Zabczyk [5] gives that this stochastic integral defines a continuous process in $H$ as soon as

$$
\int_{0}^{t} \operatorname{Tr}\left[A^{\frac{1}{2}-\frac{\varepsilon}{2}} e^{-(t-s) A} A^{\varepsilon} \mathcal{O} A^{\varepsilon} e^{-(t-s) A} A^{\frac{1}{2}-\frac{\varepsilon}{2}}\right] d s
$$

is finite (it requires easy arguments to be properly defined in spite of the unbounded operators). A sufficient condition is

$$
\int_{0}^{t}\left\|A^{\frac{1}{2}-\frac{\varepsilon}{2}} e^{-(t-s) A}\right\|_{L(H)}^{2} \operatorname{Tr}\left[A^{\varepsilon} \mathcal{O} A^{\varepsilon}\right] d s<\infty .
$$

Since

$$
\left\|A^{\frac{1}{2}-\frac{\varepsilon}{2}} e^{-(t-s) A}\right\|_{L(H)}^{2} \leq C \frac{1}{(t-s)^{1-\varepsilon}},
$$

it is sufficient to assume (A.8). It is implicitly required that $\mathcal{O}$ maps $H$ into $D\left(A^{\varepsilon}\right)$, so the operator $A^{\varepsilon} \mathcal{O} A^{\varepsilon}$ is well defined on $D\left(A^{\varepsilon}\right)$; in addition it is required that such an operator has a (unique by density) bounded extension to $H$, which has to be of trace class. Hence $z \in C\left([0, T] ; D\left(A^{\frac{1}{2}+\frac{\varepsilon}{2}}\right)\right)$, P-a.s.

Let us specialise to the case when

$$
\mathcal{O}=A^{-\gamma}
$$

In such a case, $A^{\varepsilon} \mathcal{O} A^{\varepsilon}=A^{2 \varepsilon-\gamma}$. Recall that the operator $A^{-\frac{d}{4}-\varepsilon}$ is HilbertSchmidt in $H$, if $d$ is the space dimension (it corresponds to the Sobolev embedding $\left.H^{\frac{d}{2}+2 \varepsilon}(D) \subset C(D)\right)$. Since $A^{2 \varepsilon-\gamma}$ is of trace class if $A^{\varepsilon-\frac{\gamma}{2}}$ is Hilbert-Schmidt, it can be concluded that $\varepsilon-\frac{\gamma}{2}<-\frac{3}{4}$ implies that $A^{2 \varepsilon-\gamma}$ is of trace class. Collecting all these facts, we get

Proposition A.6. If $\mathcal{O}=A^{-\frac{3}{2}-\varepsilon}$ for some $\varepsilon>0$, then

$$
z \in C\left([0, T] ; D\left(A^{\frac{1}{2}+\frac{\varepsilon}{2}}\right)\right) \quad \mathbf{P} \text {-a.s. }
$$

If $\mathcal{O}=A^{-2-\varepsilon}$ for some $\varepsilon>0$, then

$$
z \in C\left([0, T] ; D\left(A^{\frac{3}{4}+\frac{\varepsilon}{2}}\right)\right) \quad \mathbf{P} \text {-a.s. }
$$

Remark A.7. The conditions obtained in this section and in the previous one coincide. For example, assume (A.9). Indeed, first the Wiener measure gives probability one to $\left(\frac{1}{2}-\varepsilon\right)$-Hölder continuous trajectories, for arbitrarily small $\varepsilon>0$. Second, a cylindrical Wiener process in $H$ is a stochastic process with values in $D\left(A^{-\frac{3}{4}-\frac{\varepsilon}{2}}\right)$, since the embedding of $H$ into such a space is Hilbert-Schmidt. Therefore a typical trajectory of $W_{t}$ is a function of the form $A^{\frac{3}{4}+\frac{\varepsilon}{2}} h(t)$, with $h \in C^{\frac{1}{2}-\varepsilon}([0, T] ; H)$. It follows that, under the assumptions of the last corollary, a typical trajectory of 
$\sqrt{\mathcal{O}} W_{t}$ is a function of the form $A^{-1-\varepsilon} A^{\frac{3}{4}+\frac{\varepsilon}{2}} h(t)$, with $h \in C^{\frac{1}{2}-\varepsilon}([0, T] ; H)$, i.e., a function

$$
g \in C^{\frac{1}{2}-\varepsilon}\left([0, T] ; D\left(A^{\frac{1}{4}+\frac{\varepsilon}{2}}\right)\right) .
$$

\section{ACKNOWLEDGEMENTS}

We wish to thank the referee for helpful remarks and suggestions, that allowed us to clarify the presentation of the paper.

\section{REFERENCES}

1. J. Bell, D. Marcus, Vorticity intensification and the transition to turbulence in the threedimensional Euler equation, Comm. Math. Phys. 147 (1992), 371-394. MR 93c:76048

2. L. Caffarelli, R. Kohn, L. Nirenberg, Partial regularity of suitable weak solutions of the Navier-Stokes equations, Comm. Pure Appl. Math. XXXV (1982), 771-831. MR 84m:35097

3. A. J. Chorin, The evolution of a turbulent vortex, Comm. Math. Phys. 83 (1982), 517-535. MR 83g:76042

4. A. J. ChORIN, Vorticity and Turbulence, Springer-Verlag, New York 1994. MR 95m:76043

5. G. Da Prato, J. Zabczyk, Stochastic equations in infinite dimension, Cambridge Univ. Press, Cambridge 1992. MR 95g:60073

6. G. David, S. Semmes, Fractured fractals and broken dreams, Oxford Lecture Series in Mathematics and its applications 7, Clarendon Press, Oxford 1997. MR 99h:28018

7. F. Flandoli, Stochastic differential equations in fluid dynamics, Rend. Sem. Mat. Fis. Milano 66 (1996), 121-148. MR 99h:35238.

8. F. Flandoli, Irreducibility of the 3-D stochastic Navier-Stokes equation, J. Funct. Anal. 149 (1997), 160-177. MR 98j:35195

9. F. Flandoli, M. Romito, Statistically stationary solutions to the 3D Navier-Stokes equations do not show singularities, Elec. J. Prob. 6 (2001), no. 5 (electronic)

10. G. Gallavotti, Ipotesi per una introduzione alla Meccanica dei Fluidi, Quaderni del CNRGNFM 52, Roma 1996.

11. R. Grauer, T. Sideris, Numerical computation of 3D incompressible ideal fluids with swirl, Phys. Rev. Lett. 67 (1991), 3511-3514.

12. R. KerR, Evidence for a singularity of the three dimensional incompressible Euler equation, Phys. Fluids A 6 (1993), 1725-1746. MR 94d:76015

13. A. N. Kolmogorov, Local structure of turbulence in an incompressible fluid at a very high Reynolds number, Dokl. Akad. Nauk SSSR 30 (1941), 299-302; English transls., C.R. (Dokl.) Acad. Sci. URSS 30 (1941), 301-305, and Proc. Roy. Soc. London Ser. A 434 (1991), 9-13. MR 2:327d; MR 92h:76049

14. O. E. LANFord III, Time evolution of large classical systems, in Dynamical systems, theory and applications, Lecture Notes in Physics, Vol. 38, 1-111, Springer Verlag, Berlin 1975. MR 57:18653

15. J. Leray, Essai sur le mouvement d'un liquide visqueux emplissant l'espace, Acta Math. 63 (1934), 193-248.

16. B. Mandelbrot, Les objects fractals, Flammarion, Paris 1975. MR 57:2015

17. B. MandelBrot, Intermittent turbulence and fractal dimension kurtosis and the spectral exponent $\frac{5}{3}+B$, in Turbulence and Navier-Stokes equations, Lecture Notes in Math. 565, Springer-Verlag, Berlin New York Heidelberg 1976. MR 58:14335

18. J. Necas, M. Ruzicka, V. Sverak, On Leray's self-similar solutions of the Navier-Stokes equations, Acta Math. 176, No. 2 (1996), 283-294. MR 97f:35165

19. M. Romito, Existence of martingale and stationary suitable weak solutions for a stochastic Navier-Stokes system, Preprint, Quad. Dip. U. Dini, Firenze (2000).

20. M. Romito, Partial regularity theory for a stochastic Navier-Stokes system, Thesis, Pisa (2000).

21. V. Scheffer, Turbulence and Hausdorff dimension, in Turbulence and Navier-Stokes equations, Lecture Notes in Math. 565, Springer-Verlag, Berlin New York Heidelberg 1976, 174183. MR 56:10405

22. V. Scheffer, Partial regularity of solutions to the Navier-Stokes equations, Pac. J. Math. 66 (1976), 535-552. MR 56:12677] 
23. V. Scheffer, Hausdorff measure and the Navier-Stokes equations, Comm. Math. Phys. 55 (1977), 97-112. MR 58:23176

24. V. Scheffer, The Navier-Stokes equations in space dimension four, Comm. Math. Phys. 61 (1978), 41-68. MR 80i:35148

25. V. Scheffer, A solution to the Navier-Stokes inequality with an internal singularity, Comm. Math. Phys. 101 (1985), 47-85. MR 87h:35273

26. V. Scheffer, Solutions to the Navier-Stokes inequality with singularities on a Cantor set, Proc. Symp. Pure Math. 44 (1986), 359-367. MR 87k:35202

27. V. SCHEFFER, Nearly one-dimensional singularities of solutions to the Navier-Stokes inequality, Comm. Math. Phys. 110 (1987), 525-551. MR 88i:35137

28. J. SERRIN, On the interior regularity of weak solutions of the Navier-Stokes equations, Arch. Rat. Mech. Anal. 9 (1962), 187-195. MR 25:346

29. J. SERrin, The initial value problem for the Navier-Stokes equations, in Nonlinear Problems (R. E. Langer ed.) University of Wisconsin Press, Madison 1963. MR 27:442

30. R. Siegmund-Schultze, On non-equilibrium dynamics of multidimensional infinite particle systems in the translation invariant case, Comm. Math. Phys. 100 (1985), 245-265. MR 87a:82014

31. V. A. Solonnikov, Estimates of the solutions of a non-stationary linearised system of NavierStokes equations, Trudy Mat. Inst. Steklov 70 (1964), 213-317; in Amer. Math. Soc. Transl. Series 275 (1968), 1-117. MR 30:1325

32. E. Stein, Singular integrals and differentiability properties of functions, Princeton University Press, Princeton 1970. MR 44:7280

33. R. Temam, The Navier-Stokes Equations, North Holland, 1977. MR 58:29439

34. R. Temam, Navier-Stokes Equations and Nonlinear Functional Analysis, SIAM, Philadelphia, 1983. MR 86f:35152

35. R. Temam, Infinite Dimensional Dynamical Systems in Mechanics and Physics, SpringerVerlag, New York, 1988. MR 89m:58056

Dipartimento di Matematica Applicata, Università di Pisa, Via Bonanno 25/B, 56126 PisA, ITALIA

E-mail address: flandoli@dma.unipi.it

Dipartimento di Matematica, Università di Firenze, Viale Morgagni 67/A, 50134 Firenze, ITALia

E-mail address: romito@math.unifi.it 